\title{
Constraining the Kilonova Rate with Zwicky Transient Facility Searches Independent of Gravitational Wave and Short Gamma-Ray Burst Triggers
}

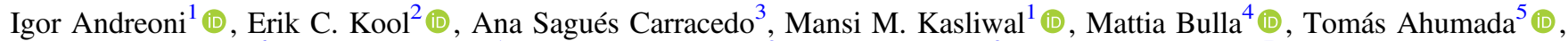 \\ Michael W. Coughlin ${ }^{6}$ (D), Shreya Anand ${ }^{1}$ (ID), Jesper Sollerman ${ }^{2}$ (10), Ariel Goobar ${ }^{3}$ (ID), David L. Kaplan ${ }^{7}$ (I), Tegan T. Loveridge ${ }^{1}$,

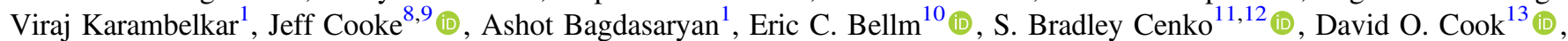 \\ Kishalay De ${ }^{1}$, Richard Dekany ${ }^{14}\left(\mathbb{D}\right.$, Alexandre Delacroix ${ }^{14}$, Andrew Drake ${ }^{1}$, Dmitry A. Duev ${ }^{1}$ (D) Christoffer Fremling $^{1}$ (D), \\ V. Zach Golkhou ${ }^{10,15}$ (D) Matthew J. Graham ${ }^{1}$, David Hale ${ }^{14}$, S. R. Kulkarni ${ }^{1}$ (D) Thomas Kupfer ${ }^{16}$ (D), Russ R. Laher ${ }^{13}$ (1),

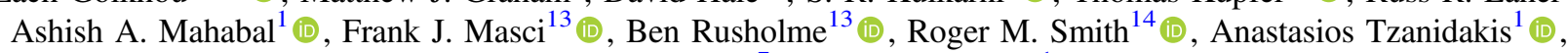 \\ Angela Van Sistine ${ }^{7}$ (D), and Yuhan $\mathrm{Yao}^{1}$ (iD \\ ${ }^{1}$ Division of Physics, Mathematics and Astronomy, California Institute of Technology, Pasadena, CA 91125, USA; andreoni@ caltech.edu \\ 2 The Oskar Klein Centre, Department of Astronomy, Stockholm University, AlbaNova, SE-106 91 Stockholm, Sweden \\ 3 The Oskar Klein Centre, Department of Physics, Stockholm University, AlbaNova, SE-106 91 Stockholm, Sweden \\ ${ }^{4}$ Nordita, KTH Royal Institute of Technology and Stockholm University, Roslagstullsbacken 23, SE-106 91 Stockholm, Sweden \\ ${ }^{5}$ Department of Astronomy, University of Maryland, College Park, MD 20742, USA \\ ${ }^{6}$ School of Physics and Astronomy, University of Minnesota, Minneapolis, MN 55455, USA \\ ${ }^{7}$ Center for Gravitation, Cosmology, and Astrophysics, Department of Physics, University of Wisconsin-Milwaukee, P.O. Box 413, Milwaukee, WI 53201, USA \\ ${ }^{8}$ Australian Research Council Centre of Excellence for Gravitational Wave Discovery (OzGrav), Swinburne University of Technology, Hawthorn, VIC, 3122, \\ Australia \\ ${ }^{9}$ Centre for Astrophysics and Supercomputing, Swinburne University of Technology, Hawthorn, VIC, 3122, Australia \\ ${ }^{10}$ DIRAC Institute, Department of Astronomy, University of Washington, 3910 15th Avenue NE, Seattle, WA 98195, USA \\ ${ }^{11}$ Astrophysics Science Division, NASA Goddard Space Flight Center, Mail Code 661, Greenbelt, MD 20771, USA \\ 12 Joint Space-Science Institute, University of Maryland, College Park, MD 20742, USA \\ ${ }^{13}$ IPAC, California Institute of Technology, 1200 E. California Blvd, Pasadena, CA 91125, USA \\ ${ }^{14}$ Caltech Optical Observatories, California Institute of Technology, Pasadena, CA 91125, USA \\ ${ }^{15}$ The eScience Institute, University of Washington, Seattle, WA 98195, USA \\ ${ }^{16}$ Kavli Institute for Theoretical Physics, University of California, Santa Barbara, CA 93106, USA \\ Received 2020 July 31; revised 2020 September 22; accepted 2020 October 5; published 2020 November 30
}

\begin{abstract}
The first binary neutron star merger, GW170817, was accompanied by a radioactivity-powered optical/infrared transient called a kilonova. To date, no compelling kilonova has been found in all-sky optical surveys, independently of short gamma-ray burst and gravitational-wave triggers. In this work, we searched the first 23 months of the Zwicky Transient Facility (ZTF) data stream for candidate kilonovae in the form of rapidly evolving transients. We combined ZTF alert queries with forced point-spread-function photometry and nightly flux stacking to increase our sensitivity to faint and fast transients. Automatic queries yielded $>11,200$ candidates, 24 of which passed quality checks and selection criteria based on a grid of kilonova models tailored for both binary neutron star and neutron star-black hole mergers. None of the candidates in our sample was deemed a possible kilonova after thorough vetting. The sources that passed our selection criteria are dominated by Galactic cataclysmic variables. We identified two fast transients at high Galactic latitude, one of which is the confirmed afterglow of long-duration GRB 190106A, the other is a possible cosmological afterglow. Using a survey simulation code, we constrained the kilonova rate for a range of models including top-hat, linearly decaying light curves, and synthetic light curves obtained with radiative transfer simulations. For prototypical GW170817-like kilonovae, we constrain the rate to be $R<1775 \mathrm{Gpc}^{-3} \mathrm{yr}^{-1}$ (95\% confidence). By assuming a population of kilonovae with the same geometry and composition of GW170817 observed under a uniform viewing angle distribution, we obtained a constraint on the rate of $R<4029 \mathrm{Gpc}^{-3} \mathrm{yr}^{-1}$.
\end{abstract}

Unified Astronomy Thesaurus concepts: Neutron stars (1108); Compact objects (288); Gravitational wave sources (677); Transient sources (1851); Surveys (1671); R-process (1324); Optical observation (1169); Optical bursts (1164); Gamma-ray bursts (629); Cataclysmic variable stars (203); Gravitational wave astronomy (675)

Supporting material: data behind figure

\section{Introduction}

The study of the dynamic sky took a twist when multimessenger discoveries became reality with the discovery of astronomical transients associated with neutrinos (Hirata et al. 1987; IceCube Collaboration et al. 2018; Stein et al. 2020) or gravitational waves (GWs; Abbott et al. 2017d). The identification of an electromagnetic (EM) counterpart to the GW event GW170817 (Abbott et al. 2017d) spectacularly confirmed the predictions that short gamma-ray bursts (GRBs; e.g., Blinnikov et al. 1984; Paczynski 1986; Eichler et al. 1989;
Narayan et al. 1992; Fong \& Berger 2013) and fast optical/nearinfrared transients called "kilonovae" or "macronovae" (KNe; e.g., Li \& Paczyński 1998; Kulkarni 2005; Rosswog 2005; Metzger et al. 2010; Metzger \& Berger 2012; Barnes \& Kasen 2013; Tanaka \& Hotokezaka 2013) can be generated by binary neutron star (BNS) or neutron star-black hole (NSBH) mergers (see also Foucart 2012; Hotokezaka et al. 2013). Launched by neutron star disruption, neutron-rich ejecta host rapid neutron capture ( $r$-process) nucleosynthesis, leading to the production of heavy elements. The radioactive decay of such 
unstable heavy nuclei powers the optical/infrared KN. Recent reviews on EM emission from neutron star mergers can be found, for example, in Metzger (2019), Nakar (2019).

The observation of optical/infrared $\mathrm{KNe}$ is particularly valuable when it happens concurrently with the discovery of a compact binary merger in GWs. The nearby BNS merger GW170817 was confidently associated with the optical transient AT2017gfo (Coulter et al. 2017) and the multimessenger discovery has led to hundreds of studies addressing, for example, astrophysics of energetic phenomena (e.g., Kasliwal et al. 2017; D'Avanzo et al. 2018; Margutti et al. 2018; Beniamini et al. 2019; Salafia \& Giacomazzo 2020), fundamental physics (e.g., Abbott et al. 2019; Coughlin et al. 2019b), and cosmology (e.g., Abbott et al. 2017a; Hotokezaka et al. 2019). It is relevant for this work to note that even the optical/infrared photometric data alone carried evidence of heavy-element nucleosynthesis in the GW170817 merger ejecta (Andreoni et al. 2017; Arcavi et al. 2017; Chornock et al. 2017; Cowperthwaite et al. 2017; Díaz et al. 2017; Drout et al. 2017; Evans et al. 2017; Kasliwal et al. 2017; Pian et al. 2017; Shappee et al. 2017; Smartt et al. 2017; Tanvir et al. 2017; Troja et al. 2017; Utsumi et al. 2017; Valenti et al. 2017).

The discovery of more $\mathrm{KNe}$ in optical surveys would allow us to estimate the production of heavy elements during neutron star mergers and better constrain the neutron star merger rate (see Section 5), therefore providing tools to tackle open questions such as whether neutron star mergers are "the site" or just "a site" for heavy-element production in the universe. This topic is discussed, for example, by Rosswog et al. (2018), Kasliwal et al. (2019), Siegel et al. (2019). The possibility of using $\mathrm{KNe}$ as standardizable candles for cosmology (without any $\mathrm{GW}$ information) is also an intriguing perspective (Coughlin et al. 2020) that can be tested when a significant population of $\mathrm{KNe}$ is unveiled.

Observationally, KNe can show an early blue component likely brighter toward polar angles for the first 2-3 days after merger, followed by a redder component generated from the tidal dynamical ejecta and post-merger ejecta (e.g., Cowperthwaite et al. 2017; Kasen et al. 2017; Kawaguchi et al. 2018; Bulla et al. 2019). Regardless of the viewing angle, KNe are expected to appear dimmer and fade more quickly relative to most supernovae at optical wavelengths. Supernovae typically peak at absolute magnitudes between $\sim-17$ and $\sim-19$ and, in most cases, they fade at a rate slower than $0.3 \mathrm{mag} \mathrm{day}^{-1}$. AT2017gfo faded at a rate $>0.5$ mag day $^{-1}$ in optical bands and it was measured to have an absolute magnitude of $M_{r} \sim-16$ shortly after peak. Such characteristics make it more difficult to discover $\mathrm{KNe}$ relative to supernovae, which are brighter at peak and remain luminous for months. Moreover, recent $\mathrm{GW}$ observations indicate a rate for BNS mergers of 250-2810 $\mathrm{Gpc}^{-3} \mathrm{yr}^{-1}$ (Abbott et al. 2020), which suggests that at most a few events per year are expected to occur at distances close enough for $\mathrm{KNe}$ to be detectable in $1 \mathrm{~m}$ class optical surveys. In comparison, the rate of unobscured supernovae in the nearby universe is $\sim 28.7 \times$ $10^{3} \mathrm{Gpc}^{-3} \mathrm{yr}^{-1}$ (Smartt et al. 2009).

Several KN candidates were found during the follow-up of short GRBs (Perley et al. 2009; Berger et al. 2013; Tanvir et al. 2013; Gao et al. 2015; Jin et al. 2015, 2016, 2020; Yang et al. 2015; Troja et al. 2018; Lamb et al. 2019) as optical/infrared excesses on top of the GRB afterglow and one was found during the follow-up of GW170817/GRB170817A (e.g., Coulter et al. 2017). Although a marginal candidate was recently identified (McBrien et al. 2020), no confirmed KN has been found during optical surveys independently of GW triggers, to date.

Searches in optical time-domain survey data sets are complementary to GW follow-up searches. The third Advanced $\mathrm{LIGO} /$ Virgo observing run (O3) took place entirely within the time frame in which data used in this work were taken and it was suspended without yielding any optical (or multiwavelength) counterpart (Andreoni et al. 2019b, 2020b; Coughlin et al. 2019a; Goldstein et al. 2019; Gomez et al. 2019; Hosseinzadeh et al. 2019; Lundquist et al. 2019; Ackley et al. 2020; Antier et al. 2020; Gompertz et al. 2020; Vieira et al. 2020; Watson et al. 2020). In addition, it is possible that neutron star mergers occurred in the nearby universe during $\mathrm{O} 3$ and were missed by the GW detectors because of instrument downtime and their unisotropic antenna pattern.

Motivated by this, we use the Zwicky Transient Facility (ZTF; Bellm et al. 2019b; Graham et al. 2019) at Palomar Observatory, which surveys the northern sky primarily in $g$ and $r$ band ( $i$ band being occasionally used for specific projects) with an instantaneous field of view of $47 \mathrm{deg}^{2}$, a pixel scale of $1^{\prime \prime}$ pixel $^{-1}$, and a typical limiting magnitude of $r \sim 20.5$ in $30 \mathrm{~s}$ exposure time, to search for serendipitous $\mathrm{KNe}$. This work is based on 239,754 images acquired during the first 23 months of ZTF operations.

The paper is organized as follows: methods used to search the ZTF alert database, to perform photometry, and to select KN candidates are described in Section 2; the results of our searches are presented in Section 3; implications of our results for $\mathrm{KN}$ rates are discussed in Section 5. Section 6 completes the manuscript with a short summary and concluding remarks. A Planck Collaboration et al. (2016) cosmology is used throughout the paper.

\section{Methods}

In this section, we describe the data set that we searched for $\mathrm{KNe}$, the data mining techniques that we adopted, and our novel scheme to vet candidates.

\subsection{Data Set}

ZTF conducts a public Survey of the Northern Sky for $40 \%$ of the time allocation. Public data are acquired as $g, r$ filter pairs with a preferred cadence of three nights, with some fields in the Galactic plane or in Transiting Exoplanet Survey Satellite (Ricker et al. 2015) fields imaged with nightly cadence (for more information on ZTF goals and planning see Bellm et al. (2019a). The remaining time allocation is divided between ZTF Partnership and Caltech surveys, usually observing a smaller sky area (including the Galactic plane) at higher cadence. Data from all programs were used in our analysis. Images are processed with the ZTF real-time reduction and image-subtraction pipeline at the Infrared Processing \& Analysis Center (Masci et al. 2019), using the ZOGY algorithm for image subtraction (Zackay et al. 2016). Upon source detection, alert packets are generated and serialized in Apache Avro format, and finally distributed to community brokers (Patterson et al. 2019). 


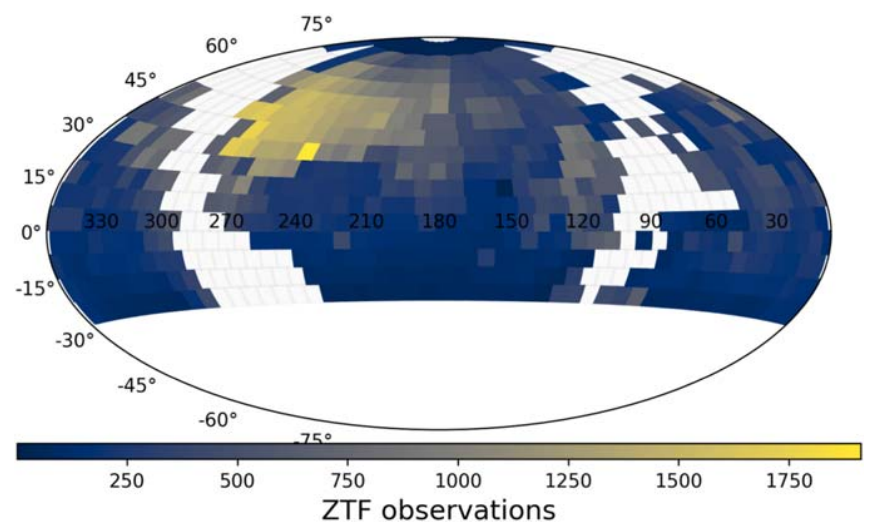

Figure 1. All-sky view of the ZTF fields searched in this work created with ztfquery (Rigault 2018), in equatorial coordinates and "hammer" projection. ZTF fields were selected to minimize the Galactic foreground by setting a threshold of $E(B-V)<0.3 \mathrm{mag}$ at their central coordinates (Planck Collaboration et al. 2014).

This work aims to discover extragalactic transients, so we limit the search for KNe to 514 fields ( $47 \mathrm{deg}^{2}$ each) with low Galactic extinction by requiring $E(B-V)<0.3$ mag at the central coordinates for the field based on Planck Collaboration et al. (2014) dust maps. The central coordinates of ZTF fields belong to two fixed grids, where the secondary grid is shifted from the primary grid in order to cover the chip gaps. We only take fields in the well-sampled ZTF primary grid, given that the secondary grid has significantly fewer observations. The effective area that we probed extended across $\sim 24,150 \mathrm{deg}^{2}$ (Figure 1). The resulting data set utilizes 239,754 images, 89,961 of which were acquired in $g$ band, 137,403 in $r$ band, and 12,390 in $i$ band.

\subsection{Alert Database Query}

The ZTF alert database was mined by querying the Kowalski ${ }^{17}$ broker at Caltech (Duev et al. 2019) in mongodb and Python. ZTF alerts are issued when a source is detected with $\geqslant 5 \sigma$ significance; however, the number of detections reported within the Avro packets (ndethist key) takes into account past detections with a lower significance, ranging between 3 and $5 \sigma$. When querying the database, we excluded those candidates that were:

1. classified with a small real/bogus score of $r b<0.5$ and deep learning scores of braai $<0.8$ and $\mathrm{drb}<0.8$, where available (Duev et al. 2019);

2. located $<10^{\prime \prime}$ from known solar system objects;

3. located $<15^{\prime \prime}$ from bright $(r<15)$ stars that often produce artificial spikes and ghost sources in their surroundings;

4. detected as "negative" flux in the image subtraction, the source having become fainter in the science image than in the reference image;

5. coincident within $2^{\prime \prime}$ radius with likely stellar sources (sgscore $>0.5$ ) found in Pan-STARRS images based on the star/galaxy separation algorithm developed by Miller et al. (2017).

We organized the experiment in two parts in order to conduct a thorough analysis on a large but manageable data set.

\footnotetext{
17 https://github.com/dmitryduev/kowalski
}

1. An all-sky search in the selected fields, requiring at least three detections in the alerts and at most 12 days time lag between the first and last detection. Such a time lag would be sufficient to observe AT2017gfo appear and fade away in our data (limiting magnitude $\sim 20.5$ ) at $40 \mathrm{Mpc}$, although $\mathrm{KNe}$ are expected to be found at larger distances, remaining bright for even less time above our detection threshold. In addition, bright $(r<18.5 \mathrm{mag})$ nearby $\mathrm{KNe}$ should have been identified in near real time during the systematic spectroscopic classification effort of the ZTF Bright Transient Survey (Fremling et al. 2020), or part of ZTF collaborative programs aiming at young/ fast transient discovery (e.g., Yao et al. 2019; Ho et al. 2020b). The query returned 10,419 candidates.

2. A galaxy-targeted search, requiring at least two detections in the alerts and at most 6 days lag between the first and last detection, with a positive crossmatch within a $100 \mathrm{kpc}$ radius (see Section 3.1) with galaxies in the Census of the Local Universe (CLU; Cook et al. 2017) catalog further than $10 \mathrm{Mpc}$. ZTF should be sensitive to faint $M_{r} \sim-9.5$ novae at $10 \mathrm{Mpc}$, thus $\mathrm{KNe}$ at that distance (or closer) are expected to appear as bright transients that our all-sky search should be able to detect easily. Faint $(r<20 \mathrm{mag}) \mathrm{KNe}$ detected several times in known galaxies closer than $200 \mathrm{Mpc}$ could have been found in near real time and classified during the Caltech volume-limited "CLU experiment" (De et al. 2020), but this experiment has not yielded any $\mathrm{KN}$ candidate, yet. This query returned 1569 candidates, 695 of which were possibly associated with galaxies $>40 \mathrm{Mpc}$ away.

Together, these searches yielded 11,202 unique candidates $(10,419$ from the all-sky search and 1569 from the galaxytargeted search, with an overlap of 786 candidates). We removed from the sample 79 sources with a counterpart in the Gaia DR2 catalog (separation $<1$ ".5) with a parallax measured to be inconsistent with $0 \pm 1.081$ mas, leaving 11,123 candidates to be further analyzed. This "stellarity" threshold was determined from the study of AllWISE catalog quasars present in the Gaia data set (Luri et al. 2018).

\subsection{Photometry and Candidate Selection Criteria}

Forced point-spread function (PSF) photometry was performed at the location of each candidate using ForcePhotZTF (Yao et al. 2019). The median coordinates recorded in the available alerts were used to improve the location accuracy. A signal-to-noise ratio of $\mathrm{S} / \mathrm{N}=3$ served as threshold for the photometric detection of a source.

First, we ran forced photometry on images taken starting a week before the first detection and until 2 weeks after the last detection of each candidate, in order to be less biased by spurious detections consistent with Gaussian noise in several thousands of images by considering the whole survey. Then, we stacked the forced photometry data points nightly in flux space to increase our sensitivity to faint sources. Finally, we ran forced photometry at the location of selected candidates for the whole duration of the survey and we rejected those candidates showing repeated activity that was not significant enough to be recorded in ZTF alerts. An example of how forced photometry and nightly stacked photometry can help reveal the behavior of faint transients is shown in Figure 2. We note that public ZTF images acquired after Public Data Release 2 on 2019 December 

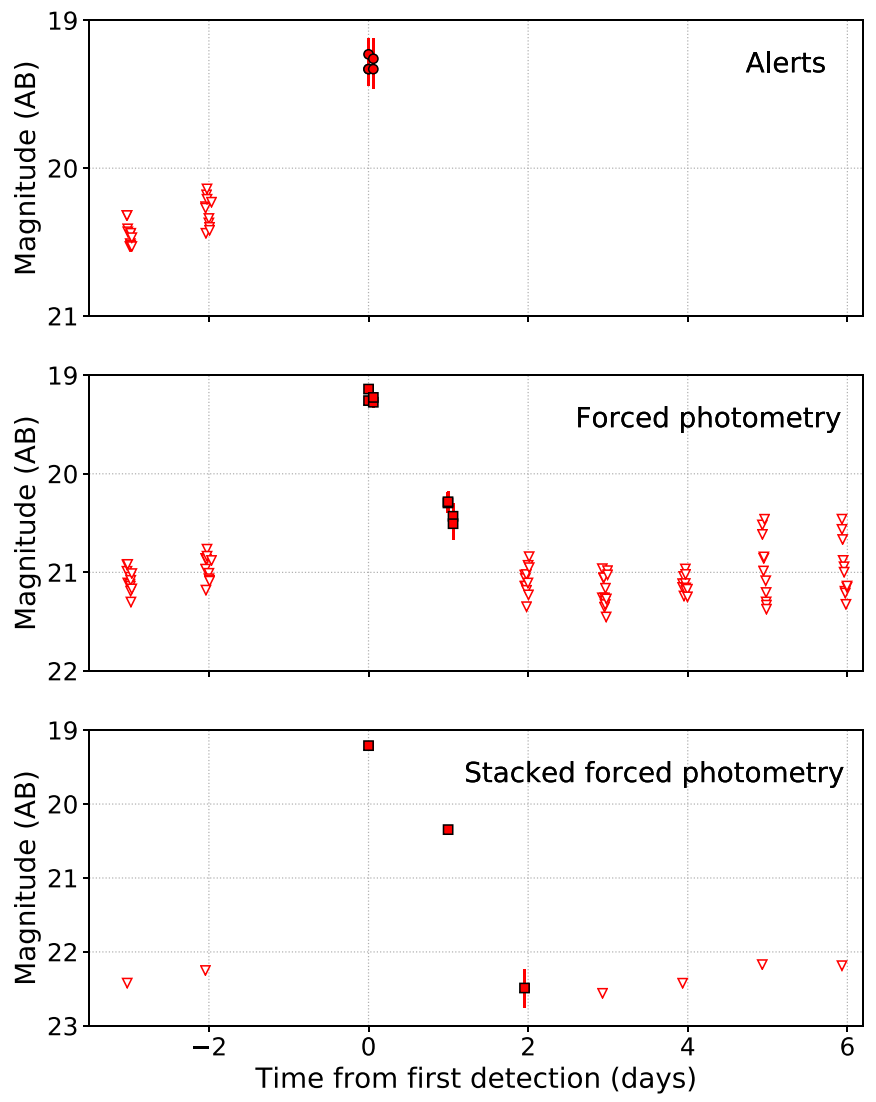

Figure 2. Light curve of the candidate ZTF19abcpiag in $r$ band built with ZTF alerts (top), forced photometry center), and nightly stacked forced photometry (bottom). The light curve based only on ZTF alerts is shallower and offers this transient detection on one night only. Single-epoch photometry with alerts or forced photometry could reveal intra-night variability, while stacked forced photometry allowed us to monitor the evolution of faint transients for a longer time. In this project we used a combination of these photometric techniques to search for KNe in ZTF data.

11 were not yet released for forced photometry when this work was carried out.

$\mathrm{KNe}$ are expected to fade rapidly, as AT2017gfo clearly confirmed. We automatically reject all those candidates that do not show any $>2 \sigma$ evolution for 6,8 , and 10 days from the first detection in $g, r$, and $i$ band, respectively. Forced photometry and nightly stacking increased the measured duration of some candidates because they allowed us to recover additional faint data points. Thus we apply another filter on stacked light curves to reject those candidates with total time span $>14$ days and with time lag between first and last detection larger than 10, 12, and 14 days in $g, r$, and $i$ band, respectively.

Where possible, we use two different linear fits before and after the brightest point to measure the rise and fade rates. In each band, we required a minimum time baseline of $3 \mathrm{hr}$ between first and last detection for the data points to be fit. If the brightest point of the light curve occurred within $3 \mathrm{hr}$ of the first or the last detection, a single linear fit was performed. Otherwise, two different linear fits were used before and after the brightest point to measure both the rise and fade rates.

Thanks to the rich optical/infrared observations available, it is tempting to use AT2017gfo as a prototypical $\mathrm{KN}$ to set the fading (or rising) thresholds to photometrically select $\mathrm{KN}$ candidates based on their photometric evolution. However, AT2017gfo is unlikely to be representative for its class, as deep follow-up and joint afterglow and $\mathrm{KN}$ fits of short GRBs have demonstrated (Gompertz et al. 2018; Ascenzi et al. 2019; Rossi et al. 2020). Instead, we chose thresholds for the fading rate based on a grid of KN models constructed with the Monte Carlo radiative transfer code POSSIS (Bulla 2019). Specifically, we use a subset of the BNS grid presented in Dietrich et al. (2020) and the NSBH grid presented in Anand et al. (2020). Both grids use geometries that are axially symmetric and constructed by varying three parameters: the mass ejected on dynamical timescales ("dynamical ejecta," $M_{\mathrm{ej}}^{\mathrm{dyn}}$ ), the mass released from the merger remnant and debris disk ("postmerger" ejecta, $\left.M_{\mathrm{ej}}^{\mathrm{pm}}\right)$, and the observer viewing angle $\left(\Theta_{\mathrm{obs}}\right.$, with $\Theta_{\text {obs }}=0^{\circ}$ corresponding to a face-on view of the system). KN light curves are predicted for 11 viewing angles from a polar $\left(\Theta_{\mathrm{obs}}=0^{\circ}\right)$ to an equatorial $\left(\Theta_{\mathrm{obs}}=90^{\circ}\right)$ orientation and for the following choices of the ejecta masses: $M_{\mathrm{ej}, \mathrm{BNS}}^{\mathrm{dyn}}=$ $[0.001,0.005,0.01,0.02] M_{\odot}, M_{\mathrm{ej}, \mathrm{BNS}}^{\mathrm{pm}} \in[0.01,0.13] M_{\odot}$ with a mass step of $0.02 M_{\odot}$, and $M_{\mathrm{ej}, \mathrm{NSBH}}^{\mathrm{dyn}}$ and $M_{\mathrm{ej}, \mathrm{NSBH}}^{\mathrm{pm}} \in$ [0.01, 0.09] $M_{\odot}$ with a mass step of $0.01 M_{\odot}$. A half-opening angle of $30^{\circ}$ is assumed for the lanthanide-rich dynamical component in both systems. This results in 308 and $891 \mathrm{KN}$ light curves for the BNS and NSBH grids, respectively. We refer the reader to Dietrich et al. (2020) and Anand et al. (2020) for more details about the adopted BNS and NSBH grids.

In Figure 3, we show the distribution of fading rates calculated between light curve peak epoch and peak time +6 days. In this work, we considered the threshold that constrains $95 \%$ of models of the grid for each filter, i.e., 0.57 , 0.39 , and 0.27 mag day $^{-1}$ for $g, r$, and $i$ band respectively. All those threshold choices fully encompass the fading rate of AT2017gfo (see blue vertical lines in Figure 3). Color evolution was not considered as a rejection criterion a priori, but it is discussed for the selected sources in Section 3.

\subsection{Candidate Vetting}

Out of 11,202 objects found when querying the ZTF alert database, 645 candidates with at least three alerts passed the selection criteria described in Section 2.3. All candidates were assessed by visually inspecting detection image triplets (made of science image, reference image, and subtraction) and light curves (individual exposures and nightly stacks). Light curves showing erratic behavior or deep upper limits in forced photometry between detections were rejected. Sequences of tens of consecutive exposures were prone to show spurious "ghost" sources that can mimic astrophysical fast transients. We effectively rejected those spurious sources aided by cone searches with Kowalski centered on the candidate's coordinates, which allowed us to flag for further inspection those candidates with several other alerts located along the readout channel direction within $20^{\prime \prime}$ from the central coordinates. This method was useful also to reject uncataloged slow-moving asteroids (in any direction, not only along the readout channel direction) that fell in our sample. 

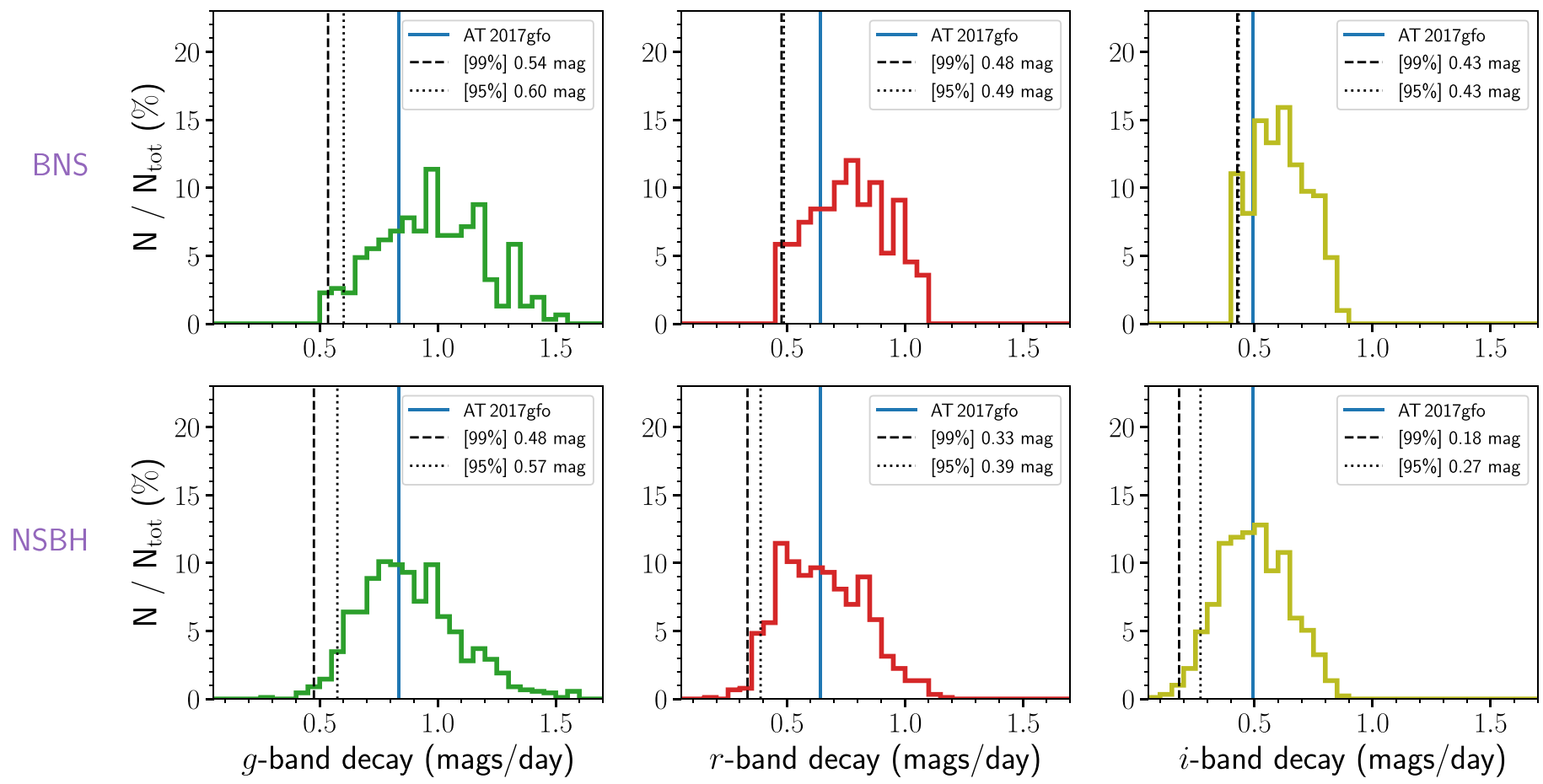

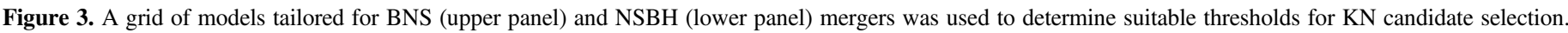

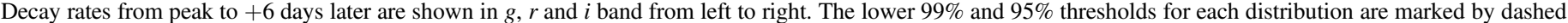

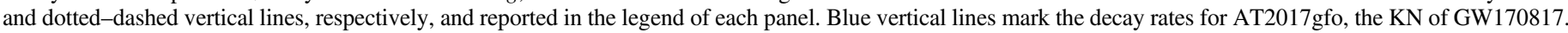

\section{Results}

In this section, we present the results of our searches, divided into an all-sky search and a galaxy-targeted search. A total of 24 sources survived the selection criteria and strict quality checks, but none of them was deemed a robust $\mathrm{KN}$ candidate. ${ }^{18}$ The main properties of the selected candidates are presented in Table 1, where candidates are grouped by (i) possible crossmatch with CLU galaxies, (ii) proximity to an extended source further than 1 !'5 and no underlying source, (iii) presence of an underlying source within 1".5, and (iv) "hostless" candidates. Appendix B presents the candidates' decay rates in each band and a detailed post mortem of individual candidates can be found in Appendix A.

\subsection{All-sky Search}

The untargeted search in 514 fields, requiring $\geqslant 3$ detections, yielded 10,419 candidates, 23 of which passed our thorough vetting. Of these, four are spatially consistent with cataloged variable sources, 10 show variability in archival Pan-STARRS 1 (PS1; Chambers et al. 2016) data or in additional ZTF data, three are likely stellar in origin, and three were rejected mainly because of the combination of color and color evolution (Figure 4) being incompatible with our grid of KN models. All the candidates rejected because of their color evolution were found at relatively low Galactic latitude $\left|b_{\text {gal }}\right| \leqslant 20 \mathrm{deg}$, which supports the hypothesis of a stellar origin. This leaves us with three candidates.

ZTF19abqtcob/AT2019aadh is located $55 \mathrm{kpc}(21 ! 88)$ from a galaxy $8.6 \mathrm{Mpc}$ away. The light curve (Figure 5) shows a rapid $r$-band decay of $\alpha_{r}=0.43$ mag day $^{-1}$, but no color

\footnotetext{
${ }^{18}$ Complete forced PSF photometry of these 24 sources from 1 week before the first detection until 3 weeks after the last detection can be found at https:// www.astro.caltech.edu/ ia/lc_candidates_IA2020_ZTF_kn_rate.csv.
}

information is available. Assuming an association with the nearby galaxy, the absolute magnitude at peak would be $M_{r} \sim-10$, consistent with classical nova luminosity. In addition, it is possible that ZTF19abqtcob is a foreground Galactic variable because of its low latitude $\left(b_{\text {gal }}=-10.0\right)$ and the lack of any other possible host galaxy visible in its proximity. Therefore, we refrain from considering ZTF19abqtcob a viable $\mathrm{KN}$ candidate.

The two remaining candidates, ZTF19aabgebm/AT2019aacx and ZTF19aanhtzz/AT2019aacu, are both located at high Galactic latitude $\left(\left|b_{\text {gal }}\right|>36\right)$ and show red $(g-r>0$ mag) color (Figure 5). ZTF19aabgebm is temporally and spatially coincident with the afterglow of the long GRB 190106A (Sonbas et al. 2019; Yurkov et al. 2019) at redshift $z=1.859$ (Schady et al. 2019). Forced photometry allowed us to calculate a decay rate for ZTF19aabgebm of $(1.24 \pm 0.26) \mathrm{mag} \mathrm{day}^{-1}$ in both $g$ and $r$ bands. An independent identification of ZTF19aabgebm in ZTF data is described in Ho et al. (2020a).

ZTF19aanhtzz could be considered the most puzzling transient found during our searches. The red color ( $g-r \sim 0.3 \mathrm{mag}$ ) disfavors the Galactic cataclysmic variable scenario, but there is no recorded gamma-ray signal temporally and spatially compatible with the optical transient to support the cosmological afterglow hypothesis. Deep $g$ - and $r$-band images were acquired with the Low Resolution Imaging Spectrometer (LRIS; Oke et al. 1995) at W. M. Keck Observatory on 2020 June 22 UT, with 600 s of exposure time (Figure 6). LRIS data were processed using lpipe (Perley 2019), a fully automatic data reduction pipeline for imaging and spectroscopy. Photometry was performed using SExtractor (Bertin \& Arnouts 2010) and it resulted in the nondetection of a source at the transient location to $3 \sigma$ limiting magnitudes of $G>26.8$ and $R>27.1$. A source is present at an angular distance of 3 ". 5 in PS1 and Legacy Survey DR8 
Table 1

Candidates that Passed Our Selection Criteria Described in Section 2.3

\begin{tabular}{|c|c|c|c|c|c|c|c|}
\hline $\begin{array}{l}\text { Name } \\
\text { Name }\end{array}$ & $\begin{array}{l}\text { R.A. } \\
\text { (deg) }\end{array}$ & $\begin{array}{l}\text { Decl. } \\
(\text { deg) }\end{array}$ & $\begin{array}{c}b_{\mathrm{gal}} \\
(\mathrm{deg})\end{array}$ & $\begin{array}{l}\text { distnr } \\
(\operatorname{arcsec})\end{array}$ & sgscore & $\begin{array}{l}\text { CLU Match } \\
\text { (Boolean) }\end{array}$ & Comment \\
\hline ZTF18aazjzed $^{\mathrm{a}}$ & 245.80994 & 65.01023 & 39.8 & $\ldots$ & $\ldots$ & 1 & Cataclysmic variable \\
\hline ZTF19abzwbxy ${ }^{\mathrm{b}}$ & 305.86048 & 6.66687 & -17.0 & $<1.5$ & 0.13 & 1 & QSO \\
\hline ZTF18achqdzh ${ }^{c}$ & 40.20850 & -9.17101 & -58.5 & $<1.5$ & 0.12 & 1 & Bright nuclear variability \\
\hline ZTF20aaouvjn ${ }^{\mathrm{b}}$ & 169.87812 & 15.41104 & 65.7 & $\cdots$ & $\ldots$ & 1 & Multiple outbursts in ZTF \\
\hline ZTF19abqtcob ${ }^{\mathrm{a}}$ & 295.23436 & 2.22770 & -10.0 & $\cdots$ & $\cdots$ & 1 & Extragalactic nova or foreground cataclysmic variable? \\
\hline ZTF18abyzkeq & 342.39259 & 37.93156 & -19.0 & 14.35 & 0.10 & 0 & Cataclysmic variable \\
\hline ZTF19aayhxlo & 295.77816 & 48.32182 & 12.0 & 8.85 & 0.06 & 0 & Post-detections in ZTF \\
\hline ZTF19acecsfi & 119.87837 & 44.27264 & 30.3 & 23.62 & 0.02 & 0 & Likely stellar/cataclysmic variable \\
\hline ZTF19aabgebm & 29.88002 & 23.84546 & -36.4 & 9.09 & 0.01 & 0 & GRB 190106A afterglow \\
\hline ZTF20aahcrjn & 109.06754 & 35.75254 & 20.2 & 9.70 & 0.03 & 0 & Multiple bursts in ZTF \\
\hline ZTF18abuzpri & 288.24987 & 61.55687 & 21.2 & $<1.5$ & 0.32 & 0 & Stellar \\
\hline ZTF19abqiwjq & 293.06491 & 6.72184 & -5.9 & $<1.5$ & 0.38 & 0 & Pre-activity in PS1 images \\
\hline ZTF19abqneae & 295.60303 & 0.56979 & -11.1 & $<1.5$ & 0.00 & 0 & Pre-activity in PS1 images \\
\hline ZTF19acbtthv & 16.02320 & 50.12632 & -12.7 & $<1.5$ & 0.49 & 0 & Pre- and post-detections in PS1 and ZTF \\
\hline ZTF19acszwgx & 271.89500 & 10.59102 & 14.5 & $<1.5$ & 0.03 & 0 & Pre-activity in PS1 images \\
\hline ZTF19abcputm & 294.13549 & -7.35038 & -13.4 & $<1.5$ & 0.14 & 0 & Pre-activity in PS1 images \\
\hline ZTF19abogdfr & 293.39693 & -17.64588 & -17.1 & $<1.5$ & 0.08 & 0 & Pre-activity in PS1 images \\
\hline ZTF19aapbbde & 270.30443 & 13.28065 & 17.0 & $<1.5$ & 0.37 & 0 & Pre-activity in PS1 images \\
\hline ZTF19abcpiag & 264.64519 & 1.42066 & 16.8 & $\cdots$ & $\cdots$ & 0 & Cataloged variable source \\
\hline ZTF18acfmhrt & 30.63920 & 55.41712 & -6.1 & $\cdots$ & $\cdots$ & 0 & Color evolution unlike $\mathrm{KN}$ \\
\hline ZTF19abxwwmr & 313.90428 & 22.98274 & -14.2 & $\cdots$ & $\cdots$ & 0 & Color evolution unlike KN \\
\hline ZTF19aanhtzz & 200.71300 & 57.46357 & 59.2 & $\cdots$ & $\cdots$ & 0 & Cosmological afterglow? \\
\hline ZTF19abudvoz & 46.68777 & 46.15358 & -10.6 & $\cdots$ & $\cdots$ & 0 & Pre-activity in PS1 images \\
\hline ZTF18acsjqjd & 29.91694 & 54.17361 & -7.4 & $\cdots$ & $\cdots$ & 0 & Pre-activity in PS1 images \\
\hline
\end{tabular}

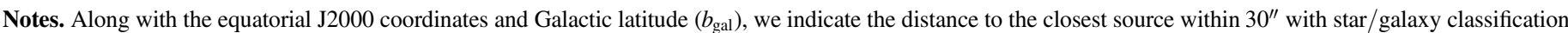

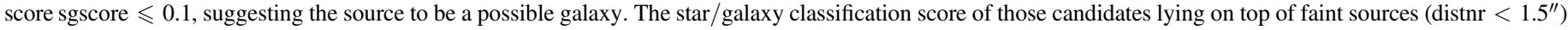

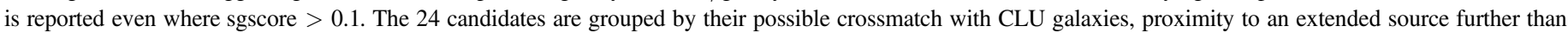

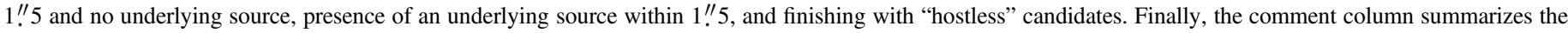
post mortem description of each candidate from Section A.

${ }^{\mathrm{a}}$ Within $100 \mathrm{kpc}$ from a galaxy $<10 \mathrm{Mpc}$ away.

${ }^{\mathrm{b}}$ Found in both all-sky and galaxy-targeted searches.

${ }^{c}$ Found only in galaxy-targeted search.

images ( $r \sim 22$, labeled as source "A" in Figure 6) and it can be modeled with a PSF shape according to the Legacy Survey DR8 catalog (Dey et al. 2019), which suggests the source to be stellar. No extended emission from source A is clearly detectable in the LRIS images.

The galaxy WISE J132245.14+572830.4, labeled as source "B" in Figure 6, is placed at a distance of $267 \mathrm{Mpc}$ by the Galaxy List for the Advanced Detector Era catalog (GLADE; Dálya et al. 2018), which would imply a projected distance of $\sim 83 \mathrm{kpc}$ between the galaxy and ZTF19aanhtzz, if it was indeed the transient's host. Such a distance is within the range of projected offsets for neutron star mergers, considering short GRBs as a proxy (Berger 2014). On 2020 July 2 UT, we acquired a spectrum of WISE J132245.14+572830.4 using the Double Spectrograph (DBSP) mounted on the Palomar 200 inch telescope with $600 \mathrm{~s}$ of exposure time. The data were processed with a custom PyRAF reduction pipeline for DBSP (Bellm \& Sesar 2016). The spectrum shows prominent Balmer series and other common galaxy features that place the galaxy at redshift $z=0.103$, i.e., at a luminosity distance of $490.57 \mathrm{Mpc}$ and a projected distance of $\sim 135 \mathrm{kpc}$ from the transient. The projected distance is too large for WISE J132245.14+572830.4 to be a reliable host of ZTF19aanhtzZ (although short GRBs were found with a projected distance from the host larger than $100 \mathrm{kpc}$; Berger 2014).
If neither source A nor source B are the transient's host, we consider the hypothesis of an underlying host galaxy being too faint to be detectable in our Keck+LRIS images. If such a faint host was located at a distance of $<250 \mathrm{Mpc}$, its luminosity would be $M \sim-10$ or fainter, typical of dwarf galaxies satellite of more massive galaxies. The apparent lack of such a massive galaxy in the field of ZTF19aanhtzz makes this scenario improbable. It is possible that a more massive host galaxy at high redshift exists that was not detectable in our Keck+LRIS images. If ZTF19aanhtzz was a Galactic source, located in the outskirts of the Milky Way, the LRIS limit suggests the absolute magnitude of the quiescent counterpart to be $M>7$ mag. Based on a typical HR diagram, this only allows a main-sequence star that is later than $\mathrm{K} 0$ in spectral type, or a white dwarf, to be the progenitor star, but an M-dwarf progenitor is likely excluded by the non-detection in $z$ band by the Legacy Survey. We consider now the scenario in which ZTF19aanhtzz was a cosmological relativistic afterglow. The lack of a detected GRB and the observation of the rise phase of the transient make it unlikely for ZTF19aanhtzz to be the afterglow of a relativistic explosion viewed on-axis. Although a detail analysis using off-axis GRB models (e.g., van Eerten et al. 2010; Beniamini et al. 2020) is outside the scope of this paper, we suggest that a slightly off-axis relativistic explosion might explain the behavior of this transient. In summary, with 


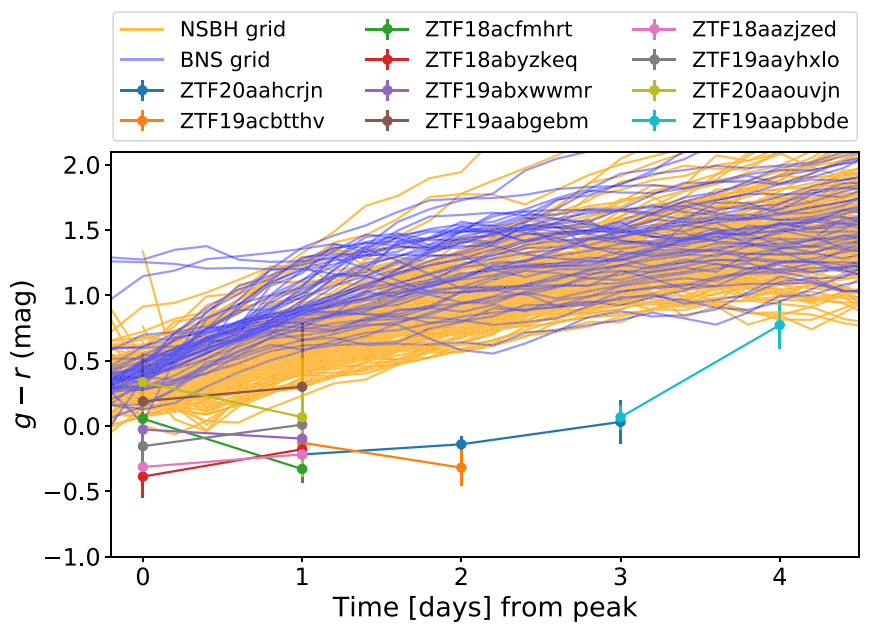

Figure 4. Here we represent the $g-r$ color evolution for BNS and NSBH grids from Bulla (2019) used in this work for the candidate selection criteria. The model light curves start (time $=0$ day) at $g$-band peak, while the candidates' phase is considered from the epoch of the brightest data point, irrespective of the filter. Galactic extinction effects are small in the selected ZTF fields and a correction (here not applied) would decrease the $g-r$ magnitude difference, moving the data points further from the $\mathrm{KN}$ models in this parameter space. The combination of color and color evolution of ZTF19aabgebm, a GRB optical afterglow, could be consistent with NSBH KN models. The behavior of other candidates for which $g-r$ information was available on multiple nights in the decline phase appears to be distant from the $\mathrm{KN}$ models considered in this work in this phase space.

the data in hand, a conclusive answer regarding the nature of ZTF19aanhtzz/AT2019aacu is yet to be found, but a cosmological afterglow origin scenario is favored.

\subsection{Galaxy-targeted Search}

In addition to all-sky searches, we conducted deeper galaxytargeted queries by requiring at least two detections (instead of three detections) to be present for each candidate. We found that 92 candidates passed the automatic selection criteria and could be spatially cross-matched with CLU galaxies $>10 \mathrm{Mpc}$ away within a $100 \mathrm{kpc}$ radius. Of those, three candidates pass the visual inspection and quality checks, namely ZTF19abzwbxy, ZTF20aaouvjn, and ZTF18achqdzh (Table 1). The former two were also found during the all-sky search (see Section 3.1), while this galaxy-targeted search yielded the new candidate ZTF18achqdzh, a bright transient $\left(M_{r}<-17\right)$ likely generated by nuclear activity. All these candidates were ruled out. We conclude that none of our candidates can be considered a viable $\mathrm{KN}$ associated with nearby $(<200 \mathrm{Mpc})$ cataloged galaxies.

\section{Discussion}

In this work, we used rigorous criteria to identify uncommon transients that could possibly be KNe. The rich ZTF data set provided us with high cadence photometry and, usually, color information that we could use to find rapidly fading candidates possibly associated with nearby galaxies and separate them from other classes of transients or variables. Our searches did not yield any source that we claim to be a viable $\mathrm{KN}$ candidate, although the nature of some fast transients remains unknown, including the possibly extragalactic fast transient ZTF19aanhtzz. It is possible that $\mathrm{KNe}$ were present in the data set, but they were missed in this analysis because, for example, they were too close to the detection limit, due to the pipeline detection efficiency, or because they evolved in different ways than we expected based on available models and on the knowledge of GW170817.

This search for KNe in the ZTF survey, independent of GW or short GRB triggers, complements the ZTF searches for optical counterparts to 13 neutron start mergers found in GWs during O3, described in Kasliwal et al. (2020). In that work, we used the query and photometry methods described in Section 2 to search for $\mathrm{KNe}$ by requiring $\geqslant 1$ detection in the alert stream over the $95 \%$ of the localization skymaps in the 3 days following the mergers. The analysis presented in Kasliwal et al. (2020) led to no viable $\mathrm{KN}$ counterpart to O3 neutron star mergers.

This work further demonstrated the importance of repeated, deep observations of wide-field surveys to better understand the nature of newly identified astronomical transients. A key problem in KN searches in "archival" survey data is the ability to recognize $\mathrm{KNe}$ without spectroscopic follow-up. The problem of photometric identification of KNe among other transients was already addressed, for example, by Cowperthwaite \& Berger (2015), Doctor et al. (2017), Cowperthwaite et al. (2018), and Bianco et al. (2019). In general, these works find that a combination of color information (at a given time) and rapid evolution is effective at separating $\mathrm{KNe}$ from other extragalactic sources such as supernovae. In our case, the selection criteria on the fading rate and transient duration had already helped us remove all supernova candidates from our sample, leaving AGN and Galactic sources as main contaminants, as expected from previous fast transient searches (e.g., van Roestel et al. 2019; Andreoni et al. 2020a).

We use the grid of models described in Section 2.3 to visualize the expected $g-r$ color evolution over time for BNS and NSBH mergers. Magnitudes and colors (specifically $g-r$ in this case) are expressed in the $\mathrm{AB}$ system. Figure 4 shows that all those candidates for which inter-night color evolution is available have color evolution different than predicted by $\mathrm{KN}$ models. Interestingly, the candidate that approaches $\mathrm{KN}$ models the most is ZTF19aabgebm, the red and rapidly evolving afterglow of GRB 190106A. We also considered a set of $30 \mathrm{KN}$ models from the grid computed by Kasen et al. (2017) with parameters similar to the blue component of AT2017gfo in the ranges $M_{\mathrm{ej}} \in[0.01,0.04] M_{\odot}, v_{k} \in[0.1-0.3]$, and $X_{\text {lan }} \in[1 \mathrm{e}-5,1 \mathrm{e}-4]$ for ejecta mass, kinetic ejecta velocity, and lanthanide fraction, respectively. In the first 3 days from the merger, these models are characterized by blue colors and thus provide better match to our $\mathrm{KN}$ candidates compared to the POSSIS grid shown in Figure 4. However, the resulting light curves get redder even more steeply than the POSSIS grid, placing the candidates even further from the region of the phase space expected for $\mathrm{KNe}$ at later epochs.

In general, systematic searches in the broadest possible parameter space covering the models presented in Section 2.3 yield reasonable fits to our selected candidates (Coughlin et al. 2018; Stachie et al. 2019); the lack of either explosion time or distance constraints make it possible to fit them within error bars of the models. This emphasizes the need for rapid photometric follow-up of sources of this type to make the most straightforward classifications.

\section{Kilonova Rates}

The searches conducted during the first 23 months of the ZTF survey, based on alerts, have not yielded any likely KN 

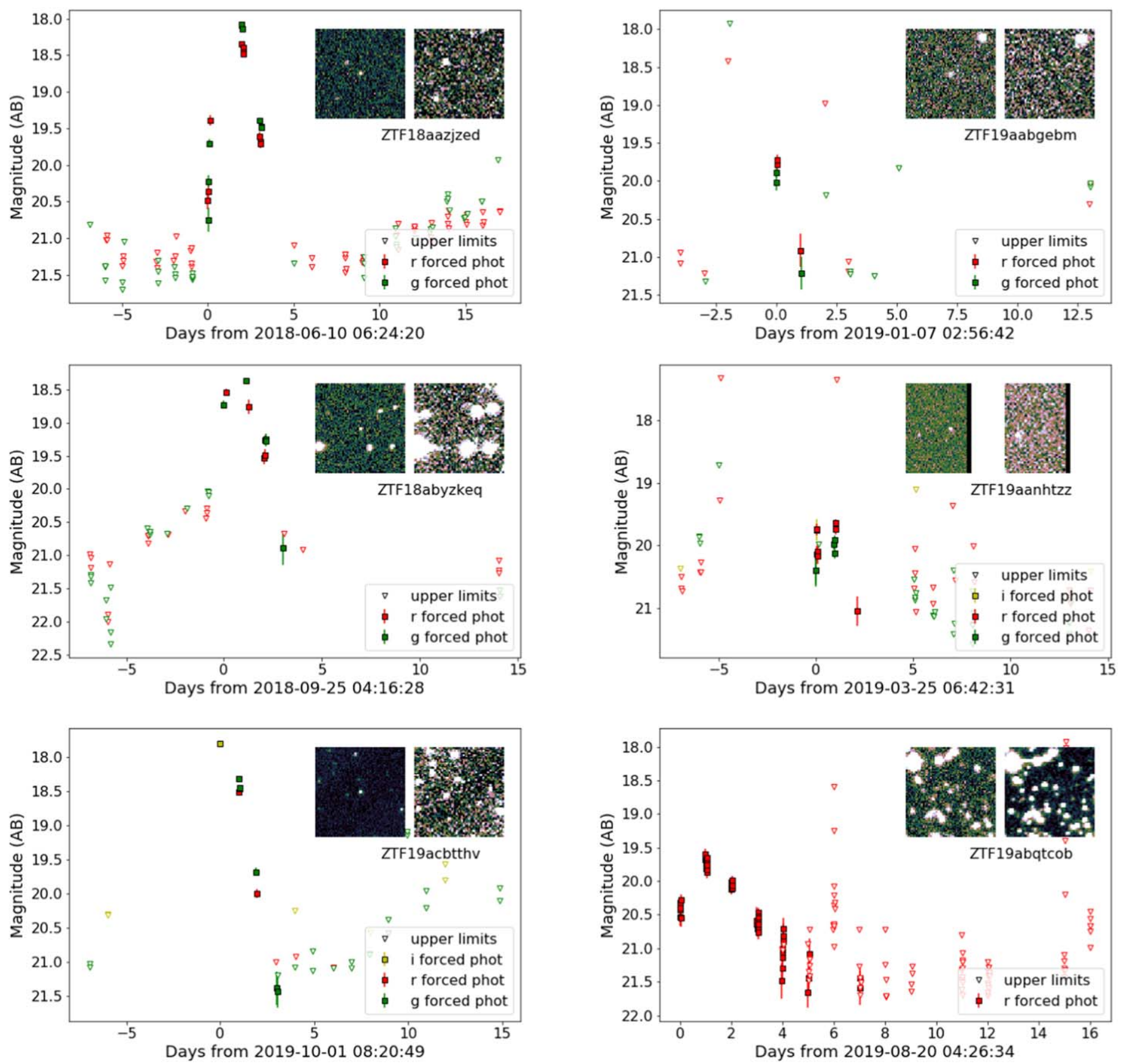

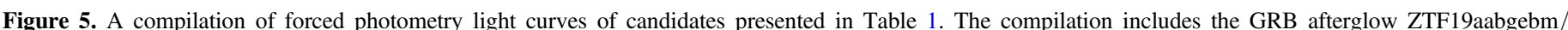

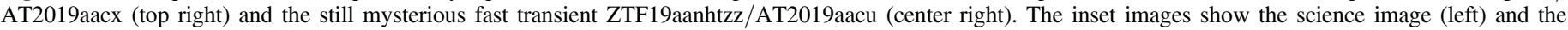
reference image (right) cutouts. The cutouts are oriented with North to the top and East to the left and have a side of $60^{\prime \prime}$.

(The data used to create this figure are available.)

candidate. This result can be used to constrain $\mathrm{KN}$ rates to compare against neutron star merger rates obtained from GW and short GRB observations.

Rates were estimated using simsurvey ${ }^{19}$ (Feindt et al. 2019), a simulation software package that accounts for custom transient light curves and observational parameters of the survey. simsurvey uses Monte Carlo methods to simulate synthetic light curves. We used ZTF data to calculate the number of $\mathrm{KNe}$ detected for each rate used in the injection of

$\overline{19}$ https://simsurvey.readthedocs.io/en/latest/ synthetic transients. Milky Way extinction was applied based on the Schlegel et al. (1998) reddening maps. We considered three types of KN light curves: a 3 days constant luminosity (or "top-hat") model, a linear decay model, and models based on radiative transport simulations. Synthetic sources are injected in simsurvey assuming a broad range of rates. Our limits on the rate are chosen at the point where three of the injected $\mathrm{KNe}$ are recovered, as dictated by Poissonian small number statistics for a 95\% confidence level (Gehrels 1986).

1. Top-hat-Constant 3 days light curve model (Appendix C). The top-hat model at $M_{g, r, i}=-16$ (about the maximum 

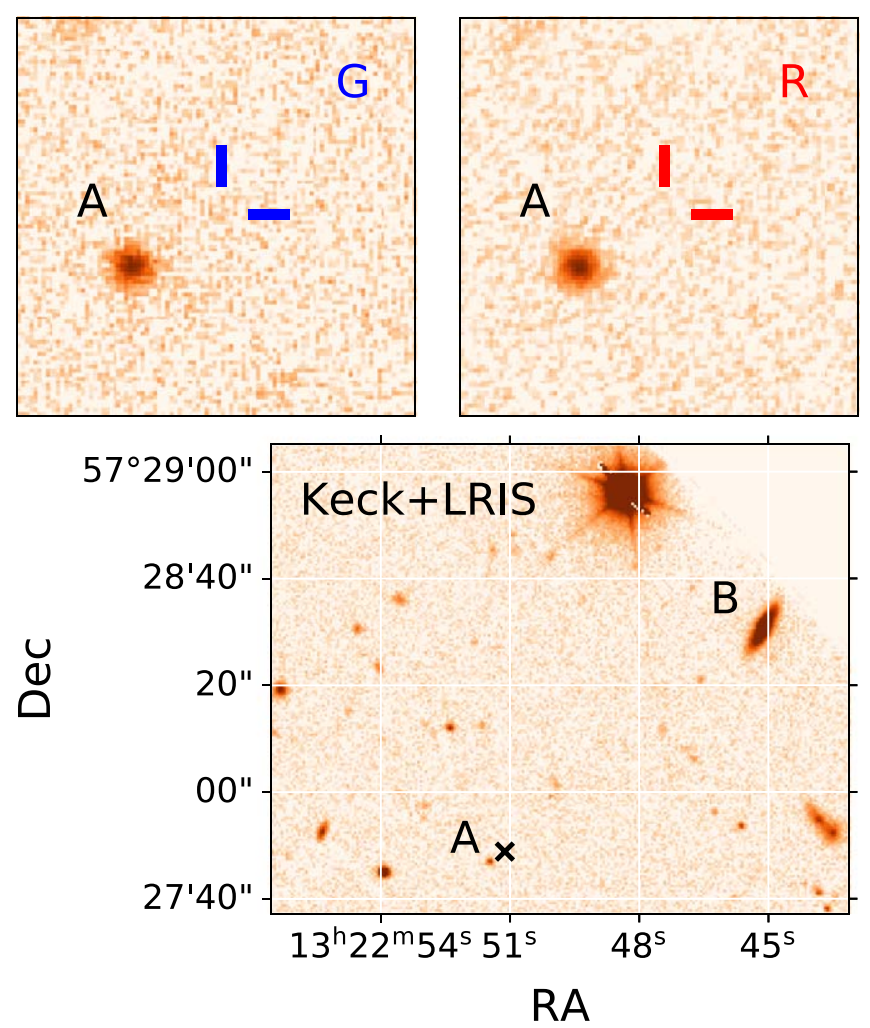

Figure 6. Keck+LRIS images of the field where ZTF19aanhtzz/AT2019aacu was found. The top panels show $g$ - and $r$-band images centered on the transient location, where the side of each squared image measures 13!. 5 . The bottom panel shows a broader view of the field, where the transient location is marked with a black cross. All figures are oriented with North to the top and East to the left. Source "A" is likely stellar and it is the closest object to ZTF19aanhtzz, with an angular separation of 3 ". 5. Source "B" is WISE J132245.14 +572830.4 , a galaxy listed in the GLADE catalog and located at an angular separation of 1!07 from ZTF19aanhtzz. Our DBSP spectrum suggests the redshift of this galaxy to be $z=0.103$, which implies a projected distance of $\sim 135 \mathrm{kpc}$, too large for a reliable host association.

brightness measured for AT2017gfo) yielded a rate of $R<398 \mathrm{Gpc}^{-3} \mathrm{yr}^{-1}$ for at least one $5 \sigma$ detection, $R<930 \mathrm{Gpc}^{-3} \mathrm{yr}^{-1}$ for at least two $5 \sigma$ detections separated by at least $3 \mathrm{hr}$, and $R<1121 \mathrm{Gpc}^{-3} \mathrm{yr}^{-1}$ by requiring at least three $5 \sigma$ detections. Appendix $\mathrm{C}$ presents a table with the results for a range of absolute magnitudes $M \in[-12,-17]$.

2. Linear decay-We considered a grid of linear decay models by varying the starting absolute magnitude $M \in[-14.5,-17.5] \mathrm{mag}$ and the decay rate $\alpha \in$ $[0.3,1.5]$ mag day $^{-1}$ to encompass the most probable decay rates that we considered to chose our selection criteria (Figure 3, Appendix D). These models do not account for color evolution and are filter-agnostic. The table in Appendix D presents the results for the linear decay grid and Figure 7 represents contours indicating rates of $R=500,1000$, and $2000 \mathrm{Gpc}^{-3} \mathrm{yr}^{-1}$.

3. Radiative transport simulations-We use the best-fit model for AT2017gfo obtained in Dietrich et al. (2020) and assume two KN populations: the first where all $\mathrm{KNe}$ have light curves like AT2017gfo, the second where KNe are instrincally like AT2017gfo, but with viewing angle $i$ uniformly distributed in $\cos (i)$. The results are shown in Appendix E. In addition, we explore the parameter space of ejecta masses for the models tailored to BNS and
NSBH from Dietrich et al. (2020) and Anand et al. (2020), also described in Section 2.3. The resulting rates for the grid of models obtained with a fixed half-opening angle $\phi=30 \mathrm{deg}$ are shown in Appendices F and G and illustrated in Figure 8. We note that the NSBH grid provides less constraining results. The light curves are fainter at peak, and broader compare to the BNS grid. Therefore, the detected light curves would be observable for longer times, but in a smaller distance range.

The rates obtained by requiring three $5 \sigma$ detections represent our all-sky search conservatively. In fact, we queried the ZTF database selecting sources with at least three detections in the alerts. However, only one alert (chronologically the last one) needs to have $\geqslant 5 \sigma$ significance. For example, it is possible that only one alert is issued for a transient when its flux passes the $5 \sigma$ threshold, but the alert packet includes information about low-significance detections (between $3 \sigma$ and $5 \sigma$ ) that did not trigger any alert generation. These low-significance detections were accounted for during our query. In addition, our experiment targeting CLU galaxies required only two detections at least $3 \mathrm{hr}$ apart, the first of which could be a low-significant detection below the $5 \sigma$ mark.

On the other hand, simsurvey does not account for the detection efficiency curve of the ZTF data processing pipeline, which is currently unavailable. Frohmaier et al. (2017) calculated the detection efficiencies for the Palomar Transient Factory (PTF) pipeline (that relies on the image-subtraction algorithm developed by Alard \& Lupton 1998), obtaining a completeness of $\sim 95 \%$ where the host galaxy surface brightness is $\lesssim 10 \%$ of the transient brightness. From the PTF experience, we can also expect the detection completeness to drop to $60 \%-70 \%$ in the presence of bright hosts with $r \lesssim 18$ mag (Frohmaier et al. 2017). However, a dedicated study of the recovery efficiency of the ZTF pipeline (that relies on the ZOGY image-subtraction algorithm; Zackay et al. 2016) is necessary to provide exact completeness figures. Such a study lies outside the scope of this paper.

We estimated that the random misalignment between science and reference images can cause a loss of $\sim 1 \%$ of the chip area. Also, Fremling et al. (2020) calculated that $<1 \%$ of astronomical transients will be lost due to proximity to bright sources outside the Galactic plane.

Catalog incompleteness should also be taken into account. A galaxy number completeness lower limit of $57 \%$ integrated between $0<z<0.05$ was obtained for the NASA/IPAC Extragalactic Database (NED) by Fremling et al. (2020) using Type Ia supernovae as proxy. The CLU catalog encompasses NED and includes $>18,000$ galaxies within $200 \mathrm{Mpc}$ found during the PTF H $\alpha$ survey (Cook et al. 2017), so we expect our integrated completeness to be larger than $57 \%$.

With these caveats in mind, we will now discuss the main results obtained in this analysis, considering the case of two $5 \sigma$ detections as the best representation of our results.

Using the best-fit model to AT2017gfo in radiative transfer simulations, for two $5 \sigma$ detections, we obtained a rate of $R<1775 \mathrm{Gpc}^{-3} \mathrm{yr}^{-1}$, while considering a population of $\mathrm{KNe}$ with the same intrinsic properties of AT2017gfo and with a uniform viewing angle distribution, we obtained a rate of $R<4029 \mathrm{Gpc}^{-3} \mathrm{yr}^{-1}$. As discussed in Section 3, models obtained with POSSIS radiative transport simulations tend to err toward redder and fainter $\mathrm{KNe}$. Assuming a blue $\mathrm{KN}$ with a linear decay rate of $\alpha=0.7$ mag day $^{-1}$ as for AT2017gfo between $g$ and $r$ band with a peak magnitude of $M=-16.5$, we can constrain the 

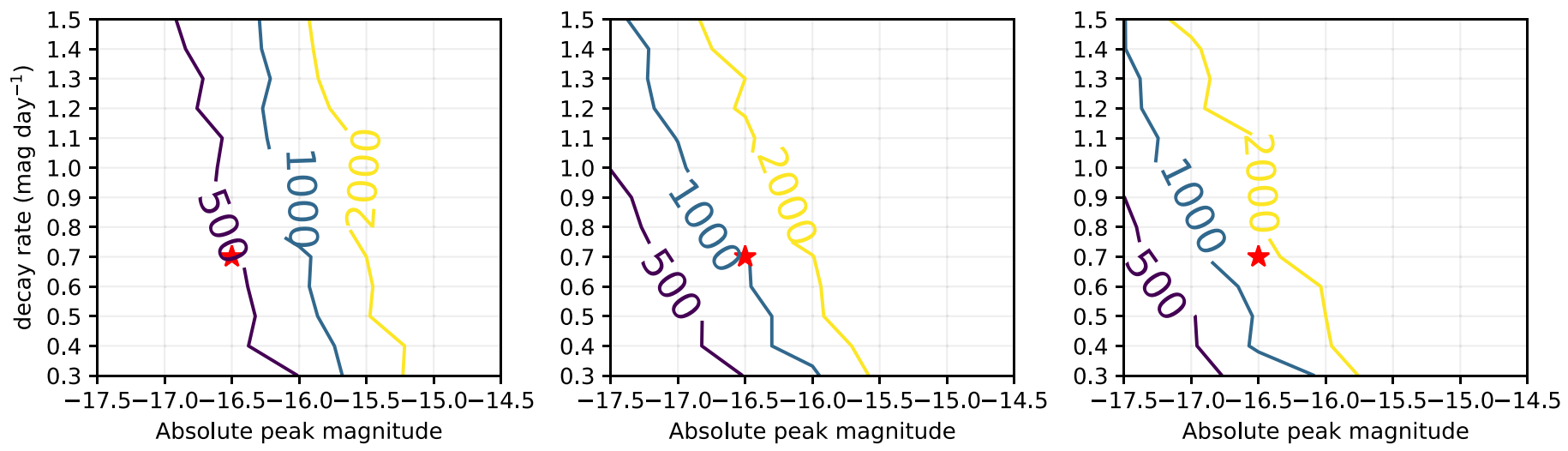

Figure 7. Estimated upper limits on the $\mathrm{KN}$ rate $\left(\mathrm{Gpc}^{-3} \mathrm{yr}^{-1}\right.$, indicated by colored contours) per peak absolute magnitude and linear decay rate requiring one (left), two (middle), and three (right) detections ( $5 \sigma$ ) for each light curve at a $95 \%$ confidence level. The red star marker indicates where a blue $\mathrm{KN}$ similar to AT2017gfo would lie if it had a starting absolute magnitude of $M=-16.5$ and a linear decay rate of 0.7 mag day ${ }^{-1}$, which is the approximate average between the measured decay rates in $g$ and $\mathrm{r}$ bands. As expected, the slower/brighter $\mathrm{KNe}$ are, the more stringent the limit on rates from ZTF is.
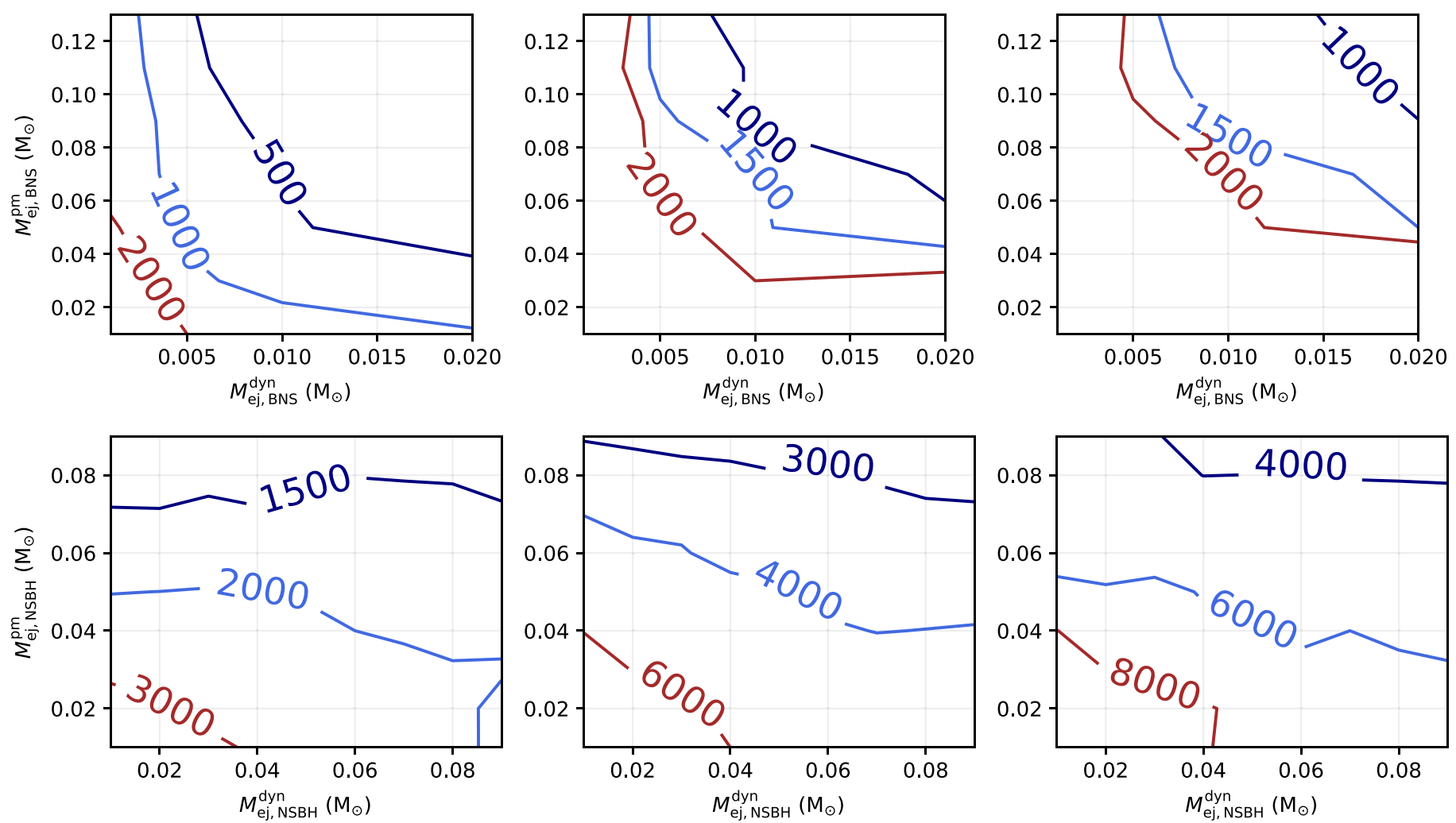

Figure 8. Estimated upper limits on the $\mathrm{KN}$ rate $\left(\mathrm{Gpc}^{-3} \mathrm{yr}^{-1}\right.$, indicated by colored contours) per ejecta mass component from BNS (top) and NSBH (bottom) grids requiring one (left), two (middle), and three (right) detections $(5 \sigma)$ for each light curve at a $95 \%$ confidence level.

$\mathrm{KN}$ rate to be $R<937 \mathrm{Gpc}^{-3} \mathrm{yr}^{-1}\left(1363 \mathrm{Gpc}^{-3} \mathrm{yr}^{-1}\right)$ by requiring two (three) detections in $\mathrm{ZTF}$ data $(5 \sigma)$.

Before the discovery of GW170817, Doctor et al. (2017) constrained the bright $\mathrm{KN}$ rate to be $R<2.4 \times 10^{4} \mathrm{Gpc}^{-3} \mathrm{yr}^{-1}$ (90\% confidence) with the Dark Energy Survey. Then, other surveys constrained the $\mathrm{KN}$ rate using AT2017gfo as reference. Smartt et al. (2017) set the $\mathrm{KN}$ rate to be $R<3.0 \times 10^{4}$ $\mathrm{Gpc}^{-3} \mathrm{yr}^{-1}$ (95\% confidence) with ATLAS and Yang et al. (2017) placed an upper limit of $R<9.9 \times 10^{4} \mathrm{Gpc}^{-3} \mathrm{yr}^{-1}(90 \%$ confidence) with 13 months of nightly monitoring of nearby galaxies with DLT40. The limits calculated in this work are more constraining than those listed above. A more stringent limit of $R<800 \mathrm{Gpc}^{-3} \mathrm{yr}^{-1}$ was placed by Kasliwal et al. (2017) with PTF data, also focusing on galaxies present in the CLU catalog (although a correction for the galaxy catalog completeness was not applied). Our results are consistent with ZTF survey simulation predictions described in Sagués Carracedo et al. (2020). The nondetection of a $\mathrm{KN}$ in almost $2 \mathrm{yr}$ of survey suggests that the number of KNe detectable with ZTF estimated by Scolnic et al. (2018) (using a fixed rate of $R=1000 \mathrm{Gpc}^{-3} \mathrm{yr}^{-1}$ and average values for the cadence, zero-point, and sky noise) may have been too optimistic.

When comparing $\mathrm{KN}$ rates with $\mathrm{BNS}$ merger or short GRB rates, a KN luminosity function should be considered, but its uncertainties are still large (Kasliwal et al. 2020). Our limits using a GW170817-like model are consistent with isotropic equivalent short GRB rates including, for example, $R=270_{-180}^{+1580}$ $\mathrm{Gpc}^{-3} \mathrm{yr}^{-1}$ (Fong et al. 2015), $R=352_{-281}^{+810} \mathrm{Gpc}^{-3} \mathrm{yr}^{-1}$ (della 


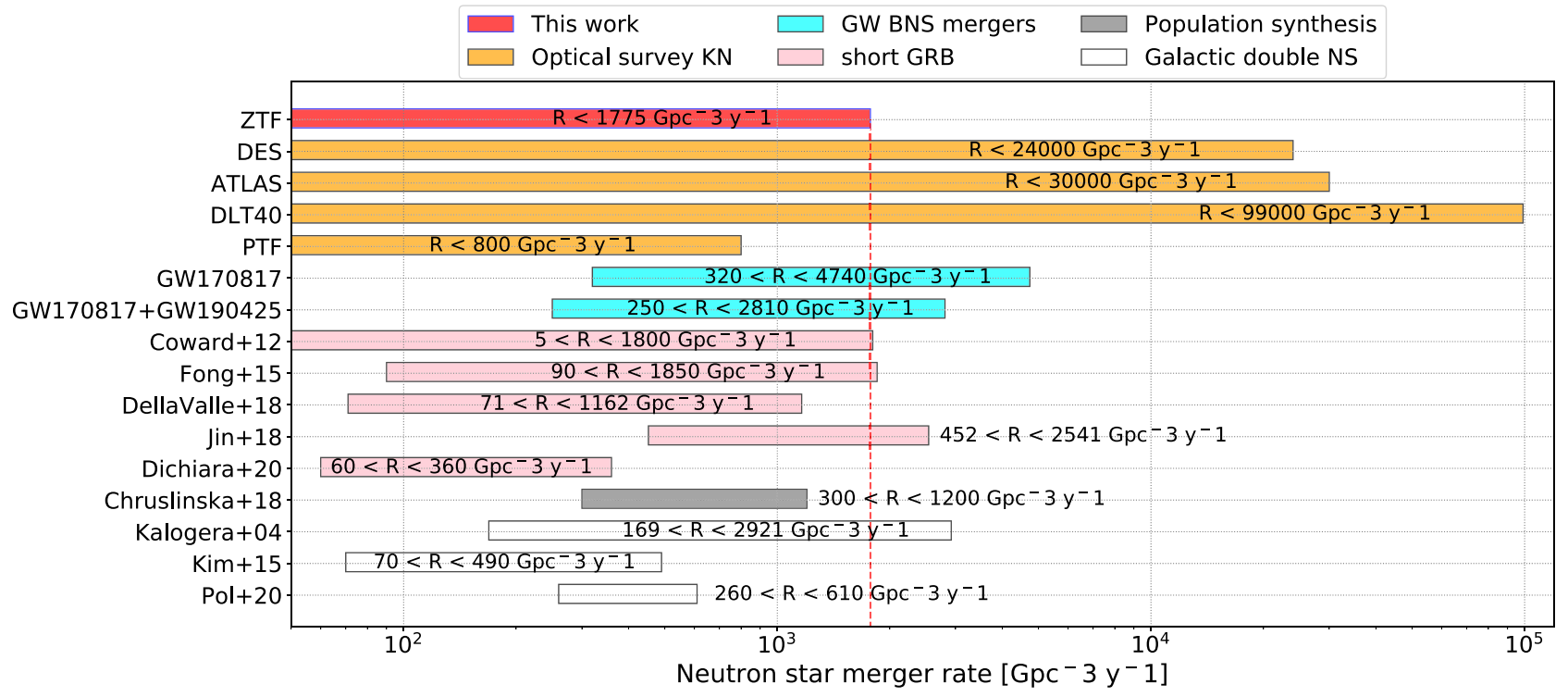

Figure 9. The constraints on the KN rate obtained in this work (red bar, dashed red line) can be compared with the results of other projects (Kasliwal et al. 2017; Smartt et al. 2017; Yang et al. 2017) that considered a uniform KN population similar to GW170817 (barred the DES result, produced before the discovery of GW170817; Doctor et al. 2017). If all BNS mergers are accompanied by KNe as bright as GW170817, then our results could improve the constraints on the BNS merger rate obtained from GW observations (Abbott et al. 2017c, 2020). In fact, the true KN luminosity function is still poorly understood. The plot also includes some examples of neutron star merger rates from short GRBs (Coward et al. 2012; Fong et al. 2015; della Valle et al. 2018; Jin et al. 2018; Dichiara et al. 2020), population synthesis (Chruslinska et al. 2018), and BNS observations in the Milky Way (Kalogera et al. 2004; Kim et al. 2015; Pol et al. 2020) for comparison.

Valle et al. 2018), or $R=160_{-100}^{+200} \mathrm{Gpc}^{-3} \mathrm{yr}^{-1}$ (Dichiara et al. 2020). ZTF results are also consistent with rates estimated with population synthesis models such as $R=600_{-300}^{+600} \mathrm{Gpc}^{-3} \mathrm{yr}^{-1}$ (Chruslinska et al. 2018), a solution among those that returned higher rates that are consistent with the BNS merger rate from GWs (Abbott et al. 2017b, 2020) (see also Tutukov \& Yungelson 1993).

Assuming that all BNS mergers generate a bright optical transient, the ZTF limits can constrain the upper side of rates obtained via GW observations of $250-2810 \mathrm{Gpc}^{-3} \mathrm{yr}^{-1}$ (Abbott et al. 2020), under the assumption that most KNe are intrinsically similar to AT2017gfo. The ZTF KN rate can also constrain the rates of $R=1109_{-657}^{+1432} \mathrm{Gpc}^{-3} \mathrm{yr}^{-1}$ (Jin et al. 2018), calculated from short GRB observations, and $R=830_{-661}^{+2091} \mathrm{Gpc}^{-3} \mathrm{yr}^{-1}$, derived from BNS system observations in the Milky Way $\left(R_{\mathrm{BNS}}=83.0_{-66.1}^{+209.1} \mathrm{Myr}^{-1}\right.$ at $95 \%$ confidence level; Kalogera et al. 2004) assuming a galaxy number density of $\sim 10^{-2} \mathrm{Mpc}^{-3}$ as in della Valle et al. (2018). Our results are consistent with BNS merger rates obtained by Kim et al. (2015), who calculated $R_{\mathrm{BNS}}=21_{-14}^{+28} \mathrm{Myr}^{-1}$ at $95 \%$ confidence level. Similarly, our result is compatible with the updated Milky Way BNS merger rate obtained by Pol et al. $(2019,2020)$ of $R_{\mathrm{BNS}}=37_{-11}^{+24} \mathrm{Myr}^{-1}$ at $90 \%$ confidence level. The rates from Kim et al. (2015) and Pol et al. (2020) translate into $R=210_{-140}^{+280} \mathrm{Gpc}^{-3} \mathrm{yr}^{-1}$ and $R=370_{-110}^{+240} \mathrm{Gpc}^{-3} \mathrm{yr}^{-1}$, respectively, using again a galaxy number density of $10^{-2} \mathrm{Mpc}^{-3}$.

Figure 9 offers a visual representation ${ }^{20}$ of the rates discussed in this section.

If changes in the viewing angle make the $\mathrm{KN}$ rate become $R<4029 \mathrm{Gpc}^{-3} \mathrm{yr}^{-1}$ as we predicted using radiative transfer models (Bulla 2019), our ZTF limits would become unconstraining for BNS mergers. The current rate from GWs does not

\footnotetext{
${ }^{20}$ A "living" version of Figure 9 can be found at https://www.astro.caltech. edu/ ia/plot_gantt_chart_rates.pdf.
}

account for NSBH mergers, which also are expected to be accompanied by bright $\mathrm{KNe}$ under favorable conditions of mass ratios and neutron star radii, thus it could be interesting to compare the ZTF constrains with new BNS + NSBH merger rates from $\mathrm{GW}$ observations in the near future.

\section{Conclusions}

We explored 23 months of ZTF survey data searching for rapidly fading transients that could be classified as $\mathrm{KNe}$ based on their photometric evolution. In addition to ZTF alert database queries, we employed forced PSF photometry and nightly flux stacking to improve our ability of recognizing $\mathrm{KNe}$ among $>10^{4}$ candidates. All most promising transients turned out to be either Galactic foreground or cosmological background sources, leaving no viable $\mathrm{KN}$ in the sample. The observations allowed us to place constraints on the $\mathrm{KN}$ rate for a variety of radiative transport-based models, specifically $R<1775 \mathrm{Gpc}^{-3} \mathrm{yr}^{-1}$ for $\mathrm{KNe}$ similar to AT2017gfo and $R<4029 \mathrm{Gpc}^{-3} \mathrm{yr}^{-1}$ for $\mathrm{KNe}$ with the same intrinsic properties of AT2017gfo assuming a uniform viewing angle distribution.

Several ongoing surveys observe large volumes of sky at increasingly high cadence including, for example, PanSTARRS (Chambers et al. 2016), the Asteroid Terrestrialimpact Last Alert System (ATLAS ${ }^{21}$ ), and All-Sky Automated Survey for Supernovae (ASAS-SN; Shappee et al. 2014; Holoien et al. 2017), ZTF itself, and multi-facility programs such as "Deeper, Wider, Faster" (Andreoni et al. 2020a; J. Cooke et al. 2020, in preparation). Dedicated searches in these large data sets may be necessary to find $\mathrm{KNe}$ (see for example Smartt et al. 2019; McBrien et al. 2020, regarding ongoing KN searches with PS1).

Based on this study, it is clear that both increased depth and rapid follow-up of interesting candidates, as executed for example

\footnotetext{
${ }^{21} \mathrm{http}: / /$ atlas.fallingstar.com/
} 
in the ZTF follow-up of GW events (Coughlin et al. 2019a; Kasliwal et al. 2020, and Anand et al. 2020), should be prioritized to maximize the probability of a $\mathrm{KN}$ discovery. In about $2 \mathrm{yr}$, ZTF has allowed us to place tighter constraints on the $\mathrm{KN}$ rate than most other surveys, but there is room for improvement in the near future. Two more years of ZTF survey will naturally halve the rate limits that we calculated. To maximize the volume explored, depth should be prioritized over area (e.g., Ghosh et al. 2017). Nightly stacking of ZTF images (D. A. Goldstein et al. 2020, in preparation) or longer exposures may be successful avenues for $\mathrm{KN}$ discovery. The identification of $\mathrm{KN}$ candidates in real time is indisputably valuable because it can trigger rapid multi-wavelength follow-up observations, including optical/infrared spectroscopy. On the other hand, the limited availability of $8 \mathrm{~m}$ class telescopes, necessary to classify sources fainter than $\sim 21 \mathrm{mag}$, represents a challenge for discovery programs relying on deeper observations.

This work has shown that photometric monitoring combined with archival information from multiple surveys can also be very effective at separating promising $\mathrm{KN}$ candidates from other types of rapidly evolving transients. The ability to recognize $\mathrm{KNe}$ solely based on their light curves will be key during the upcoming Legacy Survey of Space and Time (LSST; Ivezić et al. 2019) at the Vera C. Rubin Observatory. Photometric KN candidate identification will be possible, in particular, if a "rolling" cadence is chosen that entails nightly multi-band imaging (Andreoni et al. 2019a). Although ongoing surveys have some potential to detect $\mathrm{KNe}$ independently of GW or GRB triggers, we expect high-cadenced LSST observations to yield many serendipitous $\mathrm{KN}$ discoveries.

We thank the anonymous referee who helped us improve the quality of the manuscript. We thank P. Nugent for reviewing the manuscript and providing us with useful feedback. We thank D. Perley for useful discussion. Based on observations obtained with the Samuel Oschin 48 inch Telescope and the 60 inch Telescope at the Palomar Observatory as part of the Zwicky Transient Facility project, a scientific collaboration among the California Institute of Technology, the Oskar Klein Centre, the Weizmann Institute of Science, the University of Maryland, the University of Washington, Deutsches Elektronen-Synchrotron, the University of Wisconsin-Milwaukee, and the TANGO Program of the University System of Taiwan. Further support is provided by the U.S. National Science Foundation under grant No. AST-1440341.

This work was supported by the GROWTH (Global Relay of Observatories Watching Transients Happen) project funded by the National Science Foundation under PIRE grant No. 1545949. GROWTH is a collaborative project among California Institute of Technology (USA), University of Maryland College Park (USA), University of Wisconsin-Milwaukee (USA), Texas Tech University (USA), San Diego State University (USA), University of Washington (USA), Los Alamos National Laboratory (USA), Tokyo Institute of Technology (Japan), National Central University (Taiwan), Indian Institute of Astrophysics (India), Indian Institute of Technology Bombay (India), Weizmann Institute of Science (Israel), The Oskar Klein Centre at Stockholm University (Sweden), Humboldt University (Germany), Liverpool John Moores University (UK) and University of Sydney (Australia).

E.C.K., A.S.C., and A.G. acknowledge support from the G.R.E. A.T research environment funded by Vetenskapsrådet, the Swedish Research Council, project number 2016-06012. E.C.K. also acknowledges support from The Wenner-Gren Foundations.
M.W.C. acknowledges support from the National Science Foundation with grant No. PHY-2010970. D.L.K. is supported by NSF grant AST-1816492. M.M.K. acknowledges generous support from the David and Lucille Packard Foundation.

We thank A. Miller for the star/galaxy classification code development for ZTF. This research has made use of the VizieR catalog access tool, CDS, Strasbourg, France (doi:10.26093/cds/ vizier). The original description of the VizieR service was published in A\&AS 143, 23. This work has made use of data from the European Space Agency (ESA) mission Gaia (https:// www.cosmos.esa.int/gaia), processed by the Gaia Data Processing and Analysis Consortium (DPAC, https://www.cosmos.esa.int/ web/gaia/dpac/consortium). Funding for the DPAC has been provided by national institutions, in particular the institutions participating in the Gaia Multilateral Agreement.

Software: Astropy (The Astropy Collaboration 2018), Pandas (pandas development team 2020), Matplotlib (Caswell et al. 2020), Kowalski (Duev et al. 2019), lpipe (Perley 2019), PostgreSQL ${ }^{22}$, PyRAF DBSP data reduction pipeline (Bellm \& Sesar 2016), ztfquery (Rigault 2018), SExtractor (Bertin \& Arnouts 2010), ForcePhotZTF (Yao et al. 2019).

\section{Appendix A \\ Kilonova Candidates Post Mortem}

We discuss here individual candidates. The main features that we consider are: (1) variable catalog crossmatches; (2) possible CLU galaxy crossmatch, or presence of a bright nearby galaxy; (3) past variability in survey data other than ZTF; (4) color evolution.

1. ZTF18aazjzed-Reported to Transient Name Server (TNS) as AT20181tl, it was cataloged as a cataclysmic variable. There are no PS1 pre-detections, but a faint source is visible in the ZTF reference image and there are recent ZTF detections (after the end date of the experiment). In addition, the color evolution is incompatible with KN models (Figure 4).

2. ZTF19abzwbxy-The transient is located $86.5 \mathrm{kpc}$ from a CLU galaxy 67.2 Mpc away. However, it is coincindent with a faint source present in the PS1 catalog, making an association with the CLU galaxy unlikely. In addition, the source is cataloged as variable in the AAVSO International Variable Star Index, possibly being a quasi-stellar object (QSO; Brescia et al. 2015).

3. ZTF18achqdzh-Nuclear source located in a galaxy with spectroscopic redshift $z=0.0461$, so at peak the transient is at $M_{r}<-17$, probably too bright to be a $\mathrm{KN}$ (Kasliwal et al. 2020). The host was classified as a low-ionization nuclear emission-line region (LINER) and can harbor an AGN (Cavuoti et al. 2014). Sporadic faint detections in ZTF forced photometry suggest the emission to be due to nuclear variability.

4. ZTF20aaouvjn-Reported to TNS as AT20201ru. Located $76.2 \mathrm{kpc}\left(388^{\prime \prime}\right)$ from a galaxy $40.5 \mathrm{Mpc}$ away. About 2..5 from a star, most likely to be the source if it has large proper motion. No other sources are present nearby and there are no PS1 pre-detections. The color evolution is incompatible with KN models (Figure 4).

5. ZTF19abqtcob-Reported to TNS as AT2019aadh, it is located $55 \mathrm{kpc}\left(1313^{\prime \prime}\right)$ from a CLU galaxy $8.6 \mathrm{Mpc}$ away. If the host is indeed the nearby CLU galaxy, this would imply

\footnotetext{
22 https://postgresql.org/
} 
an absolute magnitude of $M \sim-10$ at peak, consistent with a classical nova. The low Galactic latitude of $b_{\text {gal }}=-10.0$, and the lack of any other possible host nearby make it likely for ZTF19abqtcob to be a foreground Galactic cataclysmic variable.

6. ZTF18abyzkeq-Hostless source with no PS1 predetections, ZTF18abyzkeq is classified as a variable star in the AAVSO International Variable Star Index. As expected, the color evolution is incompatible with $\mathrm{KN}$ models (Figure 4).

7. ZTF19aayhxlo-Reported to TNS as AT2019aada, ZTF19aayhxlo appears to be a hostless source with no PS1 pre-detections. The color evolution is incompatible with KN models (Figure 4). Recent ZTF detections (after the end date of the experiment) suggests the source to be a variable object.

8. ZTF19acecsfi-Faint PS1 and SDSS pre-detection within $1^{\prime \prime}$. Deep Legacy Survey images suggest the presence of an underlying blue source deemed as stellar in Legacy Survey DR8 catalog (Dey et al. 2019). Despite the high Galactic latitude $b_{\text {gal }}=30.3$, we consider the source to be likely a cataclysmic variable.

9. ZTF19aabgebm-Reported to TNS as AT2019aacx, ZTF19aabgebm is the (already known) optical afterglow of long GRB 190106A (Sonbas et al. 2019; Yurkov et al. 2019) at redshift $z=1.859$ (Schady et al. 2019). We note that its color and color evolution are barely compatible with $\mathrm{KN}$ models for NSBH mergers (Figure 4).

10. ZTF20aahcrjn - Reported to TNS as AT2020atp. A faint source is present at the candidate location in the ZTF reference image. There are no PS1 pre-detections, but forced photometry revealed another possible outburst in 2018 April, suggesting this to be a Galactic variable. Moreover, the color evolution is incompatible with $\mathrm{KN}$ models (Figure 4).

11. ZTF18abuzpri-Reported to TNS as AT20181tn. A prominent source is present in the ZTF images, likely consituted of two stars (Legacy Survey DR8). Although there is no activity recorded in the PS1 catalog, the likely stellarity and the significantly blue color $g-r=-0.73 \pm 0.18$ makes it unlikely for ZTF18abuzpri to be a KN.

12. ZTF19abqiwjq-Reported to TNS as AT2019aade, it is located in a crowded field at low Galactic latitude $b_{\mathrm{Gal}}=-5.9 \mathrm{deg}$. A faint source is present in the reference image at the location of the candidate. PS1 detected this source multiple times in different filters and the flux in these detections has increased significantly (more than eight times), indicating past activity. We therefore rule out ZTF19abqiwjq as a KN candidate.

13. ZTF19abqneae-Reported to TNS as AT2019aadd, it is located in a crowded stellar field at $b_{\mathrm{Gal}}=-11.1 \mathrm{deg}$. The candidate has multiple PS1 detections on different filters and the flux in these detections has doubled, showing activity in the past.

14. ZTF19acbtthv-Reported to TNS as AT2019aacw. Hostless source, however PS1 pre-detections are present within $2^{\prime \prime}$ and recent additional ZTF detections after the nominal end of the experiment, indicating repeated activity. Moreover, the color evolution is incompatible with KN models (Figure 4).
15. ZTF19acszwgx-Previously reported to TNS by ATLAS team as AT2016hnn. An underlying faint source is visible in the ZTF reference image, with multiple PS1 detections within $1^{\prime \prime}$. The $g$-band PS1 flux varies $30 \%$ thus it was active in the past and a possible re-brightening could be detected in recent ZTF data.

16. ZTF19abcputm-Reported to TNS as AT2019aacy. A faint source is present in the ZTF reference image, and it has multiple PS1 detection within $1^{\prime \prime}$. The $r$-band PS1 flux varies $300 \%$ thus it was active in the past.

17. ZTF19abogdfr-Reported to TNS as AT2019aacz, the source shows multiple PS1 detections within $1^{\prime \prime}$. The $g$ band PS1 flux varies by a factor of 80 , thus it was active in the past.

18. ZTF19aapbbde-The source shows an underlying blue point-like source. ZTF19aapbbde has numerous PS1 detections within $1^{\prime \prime}$, with flux measurements suggesting $\sim 50 \%$ increase in its brightness. This is grater than what would be expected within the PSF flux $3 \sigma$ error, suggesting previous activity. The source shows dramatic reddening at a 3-4 days phase, which is incompatible with the expectations from KN models (Figure 4).

19. ZTF19abcpiag-Previously reported by the Gaia team to TNS as AT2017dfl, this source is a cataloged variable star listed in the AAVSO International Variable Star Index. The transient presents an extremely rapid $r$-band light curve, but no color information is available.

20. ZTF18acfmhrt-Reported to TNS as AT20181tm, the source appears to be hostless with no pre-detections in PS1. An underlying faint blue source is visible in PS1 images. The color evolution is incompatible with KN models (Figure 4).

21. ZTF19abxwwmr-Hostless and with no PS1 pre-detections. However, the color evolution is incompatible with $\mathrm{KN}$ models (Figure 4).

22. ZTF19aanhtzz-Reported to TNS as AT2019aacu. The nearest source is 3."5 away and it is probably stellar (SDSS, Legacy Survey DR8). This source presents a number of interesting features, including red color, rapid evolution, and high Galactic latitude of $b_{\text {gal }}=59.2 \mathrm{deg}$. The lack of an apparent host galaxy suggests the source to be either a luminous cosmological transient (the afterglow of a GRB that went undetected by gamma-ray telescopes?) or some type of Galactic variable, rather than a kilonova. However, the red color disfavors the Galactic scenario and a conclusive answer regarding the nature of ZTF19aanhtzz/AT2019aacu is yet to be found.

23. ZTF19abudvoz-Previously reported to TNS by the ATLAS team as AT2016ayj in 2016. Apparently orphan, it has two PS1 pre-detections within $1^{\prime \prime}$. In addition, the light curve is also unlike what is expected for $\mathrm{KNe}$, with no color evolution during the rise time and with a $g$-band decay of $\sim 1$ mag day $^{-1}$.

24. ZTF18acsjqjd-The source shows pre-activity in PS1 images and therefore we exclude that it can be a $\mathrm{KN}$.

\section{Appendix B Candidate Fading Rates}

In Table B1 we present the fading rates (mag/day) for the candidates that passed our selection criteria and quality 
Table B1

Fading Rates (mag/day) for the Candidates Presented in Table 1

\begin{tabular}{|c|c|c|c|c|c|c|c|c|c|}
\hline Name & $\alpha_{g}$ & $\alpha_{r}$ & $\alpha_{i}$ & Forced $\alpha_{g}$ & Forced $\alpha_{r}$ & Forced $\alpha_{i}$ & Stack $\alpha_{g}$ & Stack $\alpha_{r}$ & Stack $\alpha_{i}$ \\
\hline ZTF18aazjzed & 1.30 & 1.29 & nan & 1.29 & 1.27 & nan & nan & nan & nan \\
\hline ZTF19abzwbxy & 0.82 & nan & nan & 0.82 & nan & nan & 0.82 & nan & nan \\
\hline ZTF18achqdzh & nan & nan & nan & nan & 0.51 & nan & nan & nan & nan \\
\hline ZTF20aaouvjn & nan & nan & nan & nan & nan & nan & 1.49 & 1.74 & nan \\
\hline ZTF19abqtcob & nan & 0.39 & nan & nan & 0.43 & nan & nan & nan & nan \\
\hline ZTF18abyzkeq & 0.85 & 0.45 & nan & 1.29 & 0.43 & nan & nan & 0.43 & nan \\
\hline ZTF19aayhxlo & nan & nan & nan & 1.00 & 0.97 & nan & 1.00 & nan & nan \\
\hline ZTF19acecsfi & 0.98 & nan & nan & 1.03 & nan & nan & 1.04 & nan & nan \\
\hline ZTF19aabgebm & nan & nan & nan & 1.24 & 1.23 & nan & 1.24 & 1.24 & nan \\
\hline ZTF20aahcrjn & 0.85 & 0.66 & nan & 0.89 & 0.63 & nan & 0.89 & 0.63 & nan \\
\hline ZTF18abuzpri & nan & 8.89 & nan & nan & 9.09 & nan & nan & 3.50 & nan \\
\hline ZTF19abqiwjq & nan & nan & nan & nan & 0.86 & nan & nan & 0.85 & nan \\
\hline ZTF19abqneae & nan & nan & nan & nan & 1.10 & nan & nan & 1.05 & nan \\
\hline ZTF19acbtthv & 1.44 & 1.52 & nan & 1.47 & 1.51 & nan & 1.48 & 1.51 & nan \\
\hline ZTF19acszwgx & nan & 0.52 & nan & nan & 0.52 & nan & nan & 0.53 & nan \\
\hline ZTF19abcputm & nan & 0.87 & nan & nan & 0.86 & nan & nan & 0.86 & nan \\
\hline ZTF19abogdfr & nan & 0.63 & nan & nan & 0.62 & nan & nan & 0.64 & nan \\
\hline ZTF19aapbbde & 1.11 & 0.50 & nan & 1.18 & 0.40 & nan & 1.18 & 0.41 & nan \\
\hline ZTF19abcpiag & nan & nan & nan & nan & 1.14 & nan & nan & 1.23 & nan \\
\hline ZTF18acfmhrt & 1.00 & nan & nan & 0.96 & 1.26 & nan & 0.96 & 1.26 & nan \\
\hline ZTF19abxwwmr & nan & nan & nan & 0.89 & 1.05 & nan & 0.87 & 1.06 & nan \\
\hline ZTF19aanhtzz & nan & nan & nan & nan & 1.25 & nan & nan & nan & nan \\
\hline ZTF19abudvoz & nan & nan & nan & 0.91 & nan & nan & nan & nan & nan \\
\hline ZTF18acsjqjd & nan & 0.42 & nan & nan & 0.42 & nan & nan & nan & nan \\
\hline
\end{tabular}

Table C1

checks. Specifically, we show for each band the index obtained by performing a linear fit from the brightest points light curves built with alerts, forced photometry, and nightly stacked forced photometry.

\section{Appendix C}

\section{Kilonova Rate Grid for Top-hat Models}

Table C1 presents kilonova rate upper limits for 3-day constant luminosities light curves corresponding to one, two, and three detections $(5 \sigma)$ in ZTF at $95 \%$ confidence.
Grid of Kilonova Rate Upper Limits Computed for Top-hat Models

\begin{tabular}{lccc}
\hline \hline $\mathrm{M}$ & $\begin{array}{c}R(n=1) \\
\left(\mathrm{Gpc}^{-3} \mathrm{yr}^{-1}\right)\end{array}$ & $\begin{array}{c}R(n=2) \\
\left(\mathrm{Gpc}^{-3} \mathrm{yr}^{-1}\right)\end{array}$ & $\begin{array}{c}R(n=3) \\
\left(\mathrm{Gpc}^{-3} \mathrm{yr}^{-1}\right)\end{array}$ \\
\hline-17.0 & 125.0 & 266.0 & 323.0 \\
-16.5 & 229.0 & 563.0 & 705.0 \\
-16.0 & 404.0 & 949.0 & 1162.0 \\
-15.5 & 1041.0 & 2238.0 & 2586.0 \\
-15.0 & 1595.0 & 3488.0 & 4285.0 \\
-14.5 & 2830.0 & 6521.0 & 7500.0 \\
-14.0 & 8571.0 & $\mathrm{NaN}$ & $\mathrm{NaN}$ \\
-13.5 & 10000.0 & $\mathrm{NaN}$ & $\mathrm{NaN}$ \\
-13.0 & 15000.0 & 15000.0 & 15000.0 \\
-12.5 & $\mathrm{NaN}$ & $\mathrm{NaN}$ & $\mathrm{NaN}$ \\
-12.0 & $\mathrm{NaN}$ & $\mathrm{NaN}$ & $\mathrm{NaN}$ \\
\hline
\end{tabular}


Appendix D

Kilonova Rate Grid for Linear Decay Models

Table D1 presents results for a grid of linear decay models used to inject synthetic light curves in simsurvey. The columns correspond to the starting absolute magnitude, the decay rate, and the rates corresponding to one, two, and three detections $(5 \sigma)$ in ZTF at $95 \%$ confidence.

Table D1

Grid of Kilonova Rate Upper Limits Computed for Linear Decay Models

\begin{tabular}{lcccc}
\hline \hline$M$ & $\begin{array}{c}\alpha \\
\left(\mathrm{mag} \mathrm{day}^{-1}\right)\end{array}$ & $\begin{array}{c}R(n=1) \\
\left(\mathrm{Gpc}^{-3} \mathrm{yr}^{-1}\right)\end{array}$ & $\begin{array}{c}R(n=2) \\
\left(\mathrm{Gpc}^{-3} \mathrm{yr}^{-1}\right)\end{array}$ & $\begin{array}{c}R(n=3) \\
\left(\mathrm{Gpc}^{-3} \mathrm{yr}^{-1}\right)\end{array}$ \\
\hline-17.5 & 0.3 & 82 & 141 & 184 \\
-17.5 & 0.4 & 85 & 173 & 226 \\
-17.5 & 0.5 & 99 & 200 & 251 \\
-17.5 & 0.6 & 112 & 242 & 335 \\
-17.5 & 0.7 & 140 & 306 & 379 \\
-17.5 & 0.8 & 138 & 314 & 413 \\
-17.5 & 0.9 & 135 & 340 & 447 \\
-17.5 & 1.0 & 163 & 500 & 674 \\
-17.5 & 1.1 & 181 & 545 & 714 \\
-17.5 & 1.2 & 168 & 540 & 689 \\
-17.5 & 1.3 & 187 & 594 & 789 \\
-17.5 & 1.4 & 200 & 674 & 983 \\
-17.5 & 1.5 & 205 & 750 & 967 \\
-17.0 & 0.3 & 154 & 288 & 377 \\
-17.0 & 0.4 & 179 & 335 & 444 \\
-17.0 & 0.5 & 173 & 363 & 465 \\
-17.0 & 0.6 & 232 & 535 & 697 \\
-17.0 & 0.7 & 250 & 600 & 895 \\
-17.0 & 0.8 & 272 & 722 & 967 \\
-17.0 & 0.9 & 287 & 740 & 1016 \\
-17.0 & 1.0 & 317 & 895 & 1395 \\
-17.0 & 1.1 & 315 & 1016 & 1276 \\
-17.0 & 1.2 & 344 & 1250 & 1874 \\
-17.0 & 1.3 & 375 & 1333 & 1666 \\
-17.0 & 1.4 & 382 & 1250 & 1666 \\
-17.0 & 1.5 & 441 & 1764 & 2500 \\
-16.5 & 0.3 & 304 & 508 & 645 \\
-16.5 & 0.4 & 408 & 800 & 1090 \\
-16.5 & 0.5 & 422 & 740 & 1052 \\
-16.5 & 0.6 & 397 & 952 & 1132 \\
-16.5 & 0.7 & 447 & 937 & 1363 \\
-16.5 & 0.8 & 545 & 1463 & 1874 \\
-16.5 & 0.9 & 576 & 1428 & 1874 \\
-16.5 & 1.0 & 550 & 1764 & 2142 \\
-16.5 & 1.1 & 530 & 1621 & 2000 \\
-16.5 & 1.2 & 666 & 2142 & 2500 \\
-16.5 & 1.3 & 594 & 2000 & 2857 \\
-16.5 & 1.4 & 759 & 2727 & 4000 \\
-16.5 & 1.5 & 789 & 2500 & 3333 \\
-16.0 & 0.3 & 504 & 857 & 1071 \\
-16.0 & 0.4 & 779 & 1304 & 1874 \\
& & & &
\end{tabular}

Table D1

(Continued)

\begin{tabular}{|c|c|c|c|c|}
\hline$M$ & $\begin{array}{c}\alpha \\
\left(\text { mag day }^{-1}\right)\end{array}$ & $\begin{array}{c}R(n=1) \\
\left(\mathrm{Gpc}^{-3} \mathrm{yr}^{-1}\right)\end{array}$ & $\begin{array}{c}R(n=2) \\
\left(\mathrm{Gpc}^{-3} \mathrm{yr}^{-1}\right)\end{array}$ & $\begin{array}{c}R(n=3) \\
\left(\mathrm{Gpc}^{-3} \mathrm{yr}^{-1}\right)\end{array}$ \\
\hline-16.0 & 0.5 & 645 & 1395 & 2000 \\
\hline-16.0 & 0.6 & 833 & 1500 & 2068 \\
\hline-16.0 & 0.7 & 789 & 1935 & 3333 \\
\hline-16.0 & 0.8 & 1395 & 3000 & 4285 \\
\hline-16.0 & 0.9 & 983 & 2400 & 3157 \\
\hline-16.0 & 1.0 & 1276 & 5000 & 6666 \\
\hline-16.0 & 1.1 & 1428 & 4285 & 5000 \\
\hline-16.0 & 1.2 & 1395 & 4615 & 6000 \\
\hline-16.0 & 1.3 & 1304 & 5000 & 6000 \\
\hline-16.0 & 1.4 & 1304 & 3333 & 4000 \\
\hline-16.0 & 1.5 & 1304 & 5000 & 6000 \\
\hline-15.5 & 0.3 & 1276 & 2222 & 3000 \\
\hline-15.5 & 0.4 & 1200 & 2500 & 3333 \\
\hline-15.5 & 0.5 & 1935 & 5000 & 5454 \\
\hline-15.5 & 0.6 & 1935 & 5454 & 6666 \\
\hline-15.5 & 0.7 & 2000 & 5000 & 6000 \\
\hline-15.5 & 0.8 & 2222 & 5000 & 5454 \\
\hline-15.5 & 0.9 & 2400 & 6666 & 10000 \\
\hline-15.5 & 1.0 & 1764 & 8571 & 12000 \\
\hline-15.5 & 1.1 & 2142 & 5000 & 7500 \\
\hline-15.5 & 1.2 & 2727 & 12000 & 15000 \\
\hline-15.5 & 1.3 & 3750 & nan & nan \\
\hline-15.5 & 1.4 & 4615 & 20000 & nan \\
\hline-15.5 & 1.5 & 6000 & nan & nan \\
\hline-15.0 & 0.3 & 3333 & 7500 & 12000 \\
\hline-15.0 & 0.4 & 2222 & 4285 & 4615 \\
\hline-15.0 & 0.5 & 3000 & 5000 & 5454 \\
\hline-15.0 & 0.6 & 2222 & 5000 & 6666 \\
\hline-15.0 & 0.7 & 2142 & 4615 & 5000 \\
\hline-15.0 & 0.8 & 4285 & 12000 & 12000 \\
\hline-15.0 & 0.9 & 5454 & 20000 & 20000 \\
\hline-15.0 & 1.0 & 3000 & 10000 & nan \\
\hline-15.0 & 1.1 & 4615 & 10000 & 12000 \\
\hline-15.0 & 1.2 & 3750 & 12000 & 15000 \\
\hline-15.0 & 1.3 & 3750 & 15000 & 20000 \\
\hline-15.0 & 1.4 & 7500 & nan & nan \\
\hline-15.0 & 1.5 & 10000 & 10000 & nan \\
\hline-14.5 & 0.3 & 5000 & 6000 & 6000 \\
\hline-14.5 & 0.4 & 5454 & 10000 & 10000 \\
\hline-14.5 & 0.5 & 4285 & 10000 & 12000 \\
\hline-14.5 & 0.6 & 7500 & 15000 & 20000 \\
\hline-14.5 & 0.7 & 8571 & 15000 & 15000 \\
\hline-14.5 & 0.8 & 7500 & 15000 & 15000 \\
\hline-14.5 & 0.9 & 15000 & 20000 & nan \\
\hline-14.5 & 1.0 & 8571 & nan & nan \\
\hline-14.5 & 1.1 & 10000 & nan & nan \\
\hline-14.5 & 1.2 & 8571 & 15000 & 15000 \\
\hline-14.5 & 1.3 & 7500 & nan & nan \\
\hline-14.5 & 1.4 & 15000 & nan & nan \\
\hline-14.5 & 1.5 & 10000 & 10000 & 10000 \\
\hline
\end{tabular}




\section{Appendix E}

\section{Kilonova Rate for Best-fit Model AT2017gfo}

Table E1 presents kilonova rate upper limits for the best-fit model to AT2017gfo (Dietrich et al., 2020) corresponding to one, two, and three detections $(5 \sigma)$ in ZTF at $95 \%$ confidence.

Table E1

Grid of Kilonova Rate Upper Limits Computed for the Best-fit Model to AT2017gfo

\begin{tabular}{lccc}
\hline \hline $\begin{array}{l}\text { Viewing Angle } \\
(\mathrm{deg})\end{array}$ & $\begin{array}{c}R(n=1) \\
\left(\mathrm{Gpc}^{-3} \mathrm{yr}^{-1}\right)\end{array}$ & $\begin{array}{c}R(n=2) \\
\left(\mathrm{Gpc}^{-3} \mathrm{yr}^{-1}\right)\end{array}$ & $\begin{array}{c}R(n=3) \\
\left(\mathrm{Gpc}^{-3} \mathrm{yr}^{-1}\right)\end{array}$ \\
\hline $\begin{array}{l}\text { Fixed } 20^{\circ} \\
\text { Uniform distribution in }\end{array}$ & 613 & 1775 & 2400 \\
$\quad$ cosine & 1273 & 4029 & 5510 \\
\hline
\end{tabular}

Note. Assuming a fixed viewing angle of $20 \mathrm{deg}$ and then a viewing angle distribution uniform in cosine.

\section{Appendix F Kilonova Rate Ejecta Mass BNS Grid}

Table F1 presents kilonova rate upper limits in the parameter space of ejecta masses using a model grid tailored for BNS mergers with a fixed opening angle of $\phi=30 \mathrm{deg}$ (see Section 2.3 and Dietrich et al., 2020; Anand et al., in press) corresponding to one, two, and three detections $(5 \sigma)$ in ZTF at 95\% confidence.
Table F1

Grid of Kilonova Rate Upper Limits Computed for a Grid of Dynamical and Post-merger Ejecta Masses, Tailored for BNS Mergers

\begin{tabular}{|c|c|c|c|c|}
\hline $\begin{array}{l}m_{\mathrm{dyn}} \\
\left(M_{\odot}\right)\end{array}$ & $\begin{array}{l}m_{\mathrm{pm}} \\
\left(M_{\odot}\right)\end{array}$ & $\begin{array}{c}R(n=1) \\
\left(\mathrm{Gpc}^{-3} \mathrm{yr}^{-1}\right)\end{array}$ & $\begin{array}{c}R(n=2) \\
\left(\mathrm{Gpc}^{-3} \mathrm{yr}^{-1}\right)\end{array}$ & $\begin{array}{c}R(n=3) \\
\left(\mathrm{Gpc}^{-3} \mathrm{yr}^{-1}\right)\end{array}$ \\
\hline 0.001 & 0.01 & 5000 & 12000 & 14117 \\
\hline 0.001 & 0.03 & 2876 & 6562 & 9545 \\
\hline 0.001 & 0.05 & 2142 & 5384 & 8076 \\
\hline 0.001 & 0.07 & 1500 & 2957 & 4117 \\
\hline 0.001 & 0.09 & 1478 & 3230 & 4200 \\
\hline 0.001 & 0.11 & 1354 & 2727 & 3620 \\
\hline 0.001 & 0.13 & 1272 & 3230 & 4772 \\
\hline 0.005 & 0.01 & 2000 & 6000 & 11250 \\
\hline 0.005 & 0.03 & 1153 & 3396 & 4390 \\
\hline 0.005 & 0.05 & 845 & 2278 & 3050 \\
\hline 0.005 & 0.07 & 711 & 1800 & 2368 \\
\hline 0.005 & 0.09 & 666 & 1636 & 2222 \\
\hline 0.005 & 0.11 & 538 & 1304 & 1682 \\
\hline 0.005 & 0.13 & 517 & 1200 & 1636 \\
\hline 0.010 & 0.01 & 1440 & 4090 & 6428 \\
\hline 0.010 & 0.03 & 694 & 2000 & 2535 \\
\hline 0.010 & 0.05 & 520 & 1538 & 2117 \\
\hline 0.010 & 0.07 & 491 & 1525 & 1978 \\
\hline 0.010 & 0.09 & 377 & 913 & 1276 \\
\hline 0.010 & 0.11 & 378 & 957 & 1267 \\
\hline 0.010 & 0.13 & 349 & 829 & 1132 \\
\hline 0.020 & 0.01 & 1052 & 3103 & 3750 \\
\hline 0.020 & 0.03 & 590 & 2168 & 3333 \\
\hline 0.020 & 0.05 & 396 & 1129 & 1500 \\
\hline 0.020 & 0.07 & 331 & 871 & 1250 \\
\hline 0.020 & 0.09 & 269 & 711 & 1004 \\
\hline 0.020 & 0.11 & 253 & 666 & 878 \\
\hline 0.020 & 0.13 & 228 & 623 & 850 \\
\hline
\end{tabular}




\section{Appendix G}

Kilonova Rate Ejecta Mass NSBH Grid

Table G1 presents kilonova rate upper limits in the parameter space of ejecta masses using a model grid tailored for NSBH mergers with a fixed opening angle of $\phi=30 \mathrm{deg}$ (see 2.3 and Dietrich et al., 2020; Anand et al., in press) corresponding to one, two, and three detections $(5 \sigma)$ in ZTF at $95 \%$ confidence.

Table G1

Grid of Kilonova Rate Upper Limits Computed for a Grid of Dynamical and Post-merger Ejecta Masses, Tailored for NSBH Mergers

\begin{tabular}{|c|c|c|c|c|}
\hline $\begin{array}{l}m_{\mathrm{dyn}} \\
\left(M_{\odot}\right)\end{array}$ & $\begin{array}{l}m_{\mathrm{pm}} \\
\left(M_{\odot}\right)\end{array}$ & $\begin{array}{c}R(n=1) \\
\left(\mathrm{Gpc}^{-3} \mathrm{yr}^{-1}\right)\end{array}$ & $\begin{array}{c}R(n=2) \\
\left(\mathrm{Gpc}^{-3} \mathrm{yr}^{-1}\right)\end{array}$ & $\begin{array}{c}R(n=3) \\
\left(\mathrm{Gpc}^{-3} \mathrm{yr}^{-1}\right)\end{array}$ \\
\hline 0.01 & 0.01 & 4384 & 11632 & 16285 \\
\hline 0.01 & 0.02 & 3677 & 10555 & 14250 \\
\hline 0.01 & 0.03 & 2226 & 5229 & 6867 \\
\hline 0.01 & 0.04 & 2244 & 5876 & 8028 \\
\hline 0.01 & 0.05 & 1821 & 4318 & 6195 \\
\hline 0.01 & 0.06 & 1701 & 4318 & 5700 \\
\hline 0.01 & 0.07 & 1503 & 3986 & 5000 \\
\hline 0.01 & 0.08 & 1393 & 3220 & 4710 \\
\hline 0.01 & 0.09 & 1212 & 2968 & 4285 \\
\hline 0.02 & 0.01 & 3617 & 7846 & 10625 \\
\hline 0.02 & 0.02 & 3128 & 8225 & 12439 \\
\hline 0.02 & 0.03 & 2628 & 5862 & 7968 \\
\hline 0.02 & 0.04 & 2056 & 4358 & 5730 \\
\hline 0.02 & 0.05 & 1984 & 4473 & 6071 \\
\hline 0.02 & 0.06 & 1634 & 3445 & 5257 \\
\hline 0.02 & 0.07 & 1522 & 3669 & 5257 \\
\hline 0.02 & 0.08 & 1370 & 2982 & 4080 \\
\hline 0.02 & 0.09 & 1338 & 2931 & 4047 \\
\hline 0.03 & 0.01 & 3355 & 9107 & 10625 \\
\hline 0.03 & 0.02 & 2524 & 6219 & 8360 \\
\hline 0.03 & 0.03 & 2383 & 5543 & 7727 \\
\hline 0.03 & 0.04 & 2286 & 4766 & 6710 \\
\hline 0.03 & 0.05 & 2023 & 4766 & 6144 \\
\hline 0.03 & 0.06 & 1634 & 3333 & 4678 \\
\hline 0.03 & 0.07 & 1522 & 3493 & 4636 \\
\hline 0.03 & 0.08 & 1370 & 3072 & 4080 \\
\hline 0.03 & 0.09 & 1256 & 2698 & 3617 \\
\hline 0.04 & 0.01 & 2756 & 6000 & 7083 \\
\hline 0.04 & 0.02 & 2428 & 5730 & 8095 \\
\hline 0.04 & 0.03 & 2116 & 4146 & 5604 \\
\hline 0.04 & 0.04 & 2207 & 4473 & 6800 \\
\hline 0.04 & 0.05 & 2073 & 4553 & 6986 \\
\hline 0.04 & 0.06 & 1694 & 3445 & 4811 \\
\hline 0.04 & 0.07 & 1432 & 2897 & 3750 \\
\hline 0.04 & 0.08 & 1495 & 3128 & 4722 \\
\hline 0.04 & 0.09 & 1404 & 2771 & 4080 \\
\hline 0.05 & 0.01 & 2451 & 4594 & 5862 \\
\hline 0.05 & 0.02 & 2512 & 4766 & 6623 \\
\hline 0.05 & 0.03 & 2081 & 4473 & 6538 \\
\hline 0.05 & 0.04 & 2073 & 4146 & 5730 \\
\hline 0.05 & 0.05 & 1969 & 3566 & 5151 \\
\hline 0.05 & 0.06 & 1578 & 3333 & 4636 \\
\hline 0.05 & 0.07 & 1495 & 2897 & 3805 \\
\hline 0.05 & 0.08 & 1378 & 2475 & 3566 \\
\hline 0.05 & 0.09 & 1297 & 2500 & 3227 \\
\hline 0.06 & 0.01 & 2550 & 5151 & 7083 \\
\hline 0.06 & 0.02 & 2256 & 4636 & 5862 \\
\hline 0.06 & 0.03 & 2417 & 5368 & 7727 \\
\hline 0.06 & 0.04 & 2000 & 3692 & 5052 \\
\hline 0.06 & 0.05 & 1951 & 3749 & 5393 \\
\hline 0.06 & 0.06 & 1454 & 2637 & 3692 \\
\hline 0.06 & 0.07 & 1618 & 3191 & 4166 \\
\hline 0.06 & 0.08 & 1496 & 2984 & 4042 \\
\hline
\end{tabular}

Table G1

(Continued)

\begin{tabular}{|c|c|c|c|c|}
\hline $\begin{array}{l}m_{\mathrm{dyn}} \\
\left(M_{\odot}\right)\end{array}$ & $\begin{array}{l}m_{\mathrm{pm}} \\
\left(M_{\odot}\right)\end{array}$ & $\begin{array}{c}R(n=1) \\
\left(\mathrm{Gpc}^{-3} \mathrm{yr}^{-1}\right)\end{array}$ & $\begin{array}{c}R(n=2) \\
\left(\mathrm{Gpc}^{-3} \mathrm{yr}^{-1}\right)\end{array}$ & $\begin{array}{c}R(n=3) \\
\left(\mathrm{Gpc}^{-3} \mathrm{yr}^{-1}\right)\end{array}$ \\
\hline 0.06 & 0.09 & 1289 & 2467 & 3114 \\
\hline 0.07 & 0.01 & 2368 & 5113 & 6428 \\
\hline 0.07 & 0.02 & 2132 & 4054 & 5696 \\
\hline 0.07 & 0.03 & 2261 & 5294 & 7031 \\
\hline 0.07 & 0.04 & 1867 & 3629 & 5113 \\
\hline 0.07 & 0.05 & 1679 & 3284 & 4368 \\
\hline 0.07 & 0.06 & 1704 & 3600 & 5232 \\
\hline 0.07 & 0.07 & 1685 & 3191 & 4205 \\
\hline 0.07 & 0.08 & 1535 & 3169 & 4245 \\
\hline 0.07 & 0.09 & 1319 & 2777 & 3719 \\
\hline 0.08 & 0.01 & 1867 & 3879 & 5921 \\
\hline 0.08 & 0.02 & 2083 & 4205 & 5921 \\
\hline 0.08 & 0.03 & 2031 & 3939 & 6724 \\
\hline 0.08 & 0.04 & 1788 & 4239 & 6190 \\
\hline 0.08 & 0.05 & 1751 & 3453 & 4615 \\
\hline 0.08 & 0.06 & 1777 & 3404 & 4485 \\
\hline 0.08 & 0.07 & 1384 & 2472 & 3169 \\
\hline 0.08 & 0.08 & 1465 & 2760 & 3750 \\
\hline 0.08 & 0.09 & 1235 & 2346 & 3360 \\
\hline 0.09 & 0.01 & 1925 & 3564 & 4615 \\
\hline 0.09 & 0.02 & 1925 & 4235 & 6000 \\
\hline 0.09 & 0.03 & 1836 & 3600 & 4337 \\
\hline 0.09 & 0.04 & 2130 & 4615 & 6206 \\
\hline 0.09 & 0.05 & 1825 & 3692 & 5217 \\
\hline 0.09 & 0.06 & 1685 & 3082 & 3982 \\
\hline 0.09 & 0.07 & 1555 & 3134 & 4285 \\
\hline 0.09 & 0.08 & 1390 & 2709 & 3925 \\
\hline 0.09 & 0.09 & 1220 & 2320 & 3157 \\
\hline
\end{tabular}

Igor Andreoni (i) https://orcid.org/0000-0002-8977-1498 Erik C. Kool iㅏ https://orcid.org/0000-0002-7252-3877

Mansi M. Kasliwal (i) https://orcid.org/0000-0002-5619-4938

Mattia Bulla (1) https://orcid.org/0000-0002-8255-5127

Tomás Ahumada (1) https://orcid.org/0000-0002-2184-6430 Michael W. Coughlin (1) https://orcid.org/0000-00028262-2924

Shreya Anand (1) https://orcid.org/0000-0003-3768-7515 Jesper Sollerman (1) https://orcid.org/0000-0003-1546-6615 Ariel Goobar (i) https://orcid.org/0000-0002-4163-4996 David L. Kaplan (1) https://orcid.org/0000-0001-6295-2881 Jeff Cooke (1) https://orcid.org/0000-0001-5703-2108 Eric C. Bellm (1) https://orcid.org/0000-0001-8018-5348 S. Bradley Cenko (i) https://orcid.org/0000-0003-1673-970X David O. Cook (1) https://orcid.org/0000-0002-6877-7655 Richard Dekany (ib https://orcid.org/0000-0002-5884-7867 Dmitry A. Duev (10) https://orcid.org/0000-0001-5060-8733 Christoffer Fremling (1) https://orcid.org/0000-00024223-103X

V. Zach Golkhou 1 https://orcid.org/0000-0001-8205-2506 S. R. Kulkarni (i) https://orcid.org/0000-0001-5390-8563 Thomas Kupfer (10) https://orcid.org/0000-0002-6540-1484 Russ R. Laher (1) https://orcid.org/0000-0003-2451-5482 Ashish A. Mahabal (i) https://orcid.org/0000-0003-2242-0244 Frank J. Masci (i) https://orcid.org/0000-0002-8532-9395 Ben Rusholme (i) https://orcid.org/0000-0001-7648-4142 Roger M. Smith (1) https://orcid.org/0000-0001-7062-9726 Anastasios Tzanidakis (i) https://orcid.org/0000-00030484-3331 
Angela Van Sistine (10) https://orcid.org/0000-0003-4131-173X Yuhan Yao (1) https://orcid.org/0000-0001-6747-8509

\section{References}

Abbott, B. P., Abbott, R., Abbott, T. D., et al. 2019, PhRvX, 9, 011001 Abbott, B. P., Abbott, R., Abbott, T. D., et al. 2020, ApJL, 892, L3 Abbott, B. P., Abbott, R., Abbott, T. D., et al. 2017a, Natur, 551, 85 Abbott, B. P., Abbott, R., Abbott, T. D., et al. 2017b, PhRvL, 118, 221101 Abbott, B. P., Abbott, R., Abbott, T. D., et al. 2017c, PhRvL, 119, 161101 Abbott, B. P., Abbott, R., Abbott, T. D., et al. 2017d, ApJL, 848, L12 Ackley, K., Amati, L., Barbieri, C., et al. 2020, arXiv:2002.01950 Alard, C., \& Lupton, R. H. 1998, ApJ, 503, 325

Anand, S., Coughlin, M. W., Kasliwal, M. M., et al. 2020, NatAs, 2020, 179 Andreoni, I., Ackley, K., Cooke, J., et al. 2017, PASA, 34, e069 Andreoni, I., Anand, S., Bianco, F. B., et al. 2019a, PASP, 131, 068004 Andreoni, I., Cooke, J., Webb, S., et al. 2020a, MNRAS, 491, 5852 Andreoni, I., Goldstein, D. A., Anand, S., et al. 2019b, ApJL, 881, L16 Andreoni, I., Goldstein, D. A., Kasliwal, M. M., et al. 2020b, ApJ, 890, 131 Antier, S., Agayeva, S., Almualla, M., et al. 2020, MNRAS, 497, 5518 Arcavi, I., Hosseinzadeh, G., Howell, D. A., et al. 2017, Natur, 551, 64 Ascenzi, S., Coughlin, M. W., Dietrich, T., et al. 2019, MNRAS, 486, 672 Barnes, J., \& Kasen, D. 2013, ApJ, 775, 18

Bellm, E. C., Kulkarni, S. R., Barlow, T., et al. 2019a, PASP, 131, 068003 Bellm, E. C., Kulkarni, S. R., Graham, M. J., et al. 2019b, PASP, 131, 018002 Bellm, E. C., \& Sesar, B. 2016, pyraf-dbsp: Reduction Pipeline for the Palomar Double Beam Spectrograph, Astrophysics Source Code Library, ascl: 1602.002

Beniamini, P., Granot, J., \& Gill, R. 2020, MNRAS, 493, 3521

Beniamini, P., Petropoulou, M., Barniol Duran, R., \& Giannios, D. 2019, MNRAS, 483, 840

Berger, E. 2014, ARA\&A, 52, 43

Berger, E., Fong, W., \& Chornock, R. 2013, ApJL, 774, L23

Bertin, E., \& Arnouts, S. 2010, SExtractor: Source Extractor, Astrophysics Source Code Library, ascl:1010.064

Bianco, F. B., Drout, M. R., Graham, M. L., et al. 2019, PASP, 131, 068002 Blinnikov, S. I., Novikov, I. D., Perevodchikova, T. V., \& Polnarev, A. G. 1984, SvAL, 10, 177

Brescia, M., Cavuoti, S., \& Longo, G. 2015, MNRAS, 450, 3893

Bulla, M. 2019, MNRAS, 489, 5037

Bulla, M., Covino, S., Kyutoku, K., et al. 2019, NatAs, 3, 99

Caswell, T. A., Droettboom, M., Lee, A., et al. 2020, matplotlib/matplotlib: REL: v3.2.2, Zenodo, doi:10.5281/zenodo. 3898017

Cavuoti, S., Brescia, M., D’Abrusco, R., Longo, G., \& Paolillo, M. 2014, MNRAS, 437, 968

Chambers, K. C., Magnier, E. A., Metcalfe, N., et al. 2016, arXiv:1612.05560

Chornock, R., Berger, E., Kasen, D., et al. 2017, ApJL, 848, L19

Chruslinska, M., Belczynski, K., Klencki, J., \& Benacquista, M. 2018 MNRAS, 474, 2937

Cook, D. O., Kasliwal, M. M., Van Sistine, A., et al. 2017, arXiv:1710.05016 Coughlin, M. W., Ahumada, T., Anand, S., et al. 2019a, ApJL, 885, L19

Coughlin, M. W., Dietrich, T., Doctor, Z., et al. 2018, MNRAS, 480, 3871

Coughlin, M. W., Dietrich, T., Heinzel, J., et al. 2020, PhRvR, 2, 022006

Coughlin, M. W., Dietrich, T., Margalit, B., \& Metzger, B. D. 2019b, MNRAS, 489, L91

Coulter, D. A., Foley, R. J., Kilpatrick, C. D., et al. 2017, Sci, 358, 1556

Coward, D. M., Howell, E. J., Piran, T., et al. 2012, MNRAS, 425, 2668

Cowperthwaite, P. S., \& Berger, E. 2015, ApJ, 814, 25

Cowperthwaite, P. S., Berger, E., Rest, A., et al. 2018, ApJ, 858, 18

Cowperthwaite, P. S., Berger, E., Villar, V. A., et al. 2017, ApJL, 848, L17

Dálya, G., Galgóczi, G., Dobos, L., et al. 2018, MNRAS, 479, 2374

D’Avanzo, P., Campana, S., Salafia, O. S., et al. 2018, A\&A, 613, L1

De, K., Kasliwal, M. M., Tzanidakis, A., et al. 2020, arXiv:2004.09029 della Valle, M., Guetta, D., Cappellaro, E., et al. 2018, MNRAS, 481, 4355 Dey, A., Schlegel, D. J., Lang, D., et al. 2019, AJ, 157, 168

Díaz, M. C., Macri, L. M., Garcia Lambas, D., et al. 2017, ApJL, 848, L29 Dichiara, S., Troja, E., O'Connor, B., et al. 2020, MNRAS, 492, 5011

Dietrich, T., Coughlin, M. W., Pang, P. T. H., et al. 2020, arXiv:2002.11355

Doctor, Z., Kessler, R., Chen, H. Y., et al. 2017, ApJ, 837, 57

Drout, M. R., Piro, A. L., Shappee, B. J., et al. 2017, Sci, 358, 1570

Duev, D. A., Mahabal, A., Masci, F. J., et al. 2019, MNRAS, 489, 3582

Eichler, D., Livio, M., Piran, T., \& Schramm, D. N. 1989, Nat, 340, 126

Evans, P. A., Cenko, S. B., Kennea, J. A., et al. 2017, Sci, 358, 1565

Feindt, U., Nordin, J., Rigault, M., et al. 2019, JCAP, 2019, 005

Fong, W., \& Berger, E. 2013, ApJ, 776, 18
Fong, W., Berger, E., Margutti, R., \& Zauderer, B. A. 2015, ApJ, 815, 102 Foucart, F. 2012, PhRvD, 86, 124007

Fremling, C., Miller, A. A., Sharma, Y., et al. 2020, ApJ, 895, 32

Frohmaier, C., Sullivan, M., Nugent, P. E., Goldstein, D. A., \& DeRose, J. 2017, ApJS, 230, 4

Gao, H., Ding, X., Wu, X.-F., Dai, Z.-G., \& Zhang, B. 2015, ApJ, 807, 163 Gehrels, N. 1986, ApJ, 303, 336

Ghosh, S., Chatterjee, D., Kaplan, D. L., Brady, P. R., \& van Sistine, A. 2017, PASP, 129, 114503

Goldstein, D. A., Andreoni, I., Nugent, P. E., et al. 2019, ApJL, 881, L7

Gomez, S., Hosseinzadeh, G., Cowperthwaite, P. S., et al. 2019, ApJL, 884, L55

Gompertz, B. P., Cutter, R., Steeghs, D., et al. 2020, MNRAS, 497, 726

Gompertz, B. P., Levan, A. J., Tanvir, N. R., et al. 2018, ApJ, 860, 62

Graham, M., Kulkarni, S. R., Bellm, E. C., et al. 2019, PASP, 131, 078001

Hirata, K., Kajita, T., Koshiba, M., Nakahata, M., \& Oyama, Y. 1987, PhRvL, 58,1490

Ho, A. Y. Q., Perley, D. A., Beniamini, P., et al. 2020a, arXiv:2006.10761

Ho, A. Y. Q., Perley, D. A., Kulkarni, S. R., et al. 2020b, ApJ, 895, 49

Holoien, T. W.-S., Brown, J. S., Stanek, K. Z., et al. 2017, MNRAS, 471, 4966

Hosseinzadeh, G., Cowperthwaite, P. S., Gomez, S., et al. 2019, ApJL, 880, L4

Hotokezaka, K., Kyutoku, K., Tanaka, M., et al. 2013, ApJL, 778, L16

Hotokezaka, K., Nakar, E., Gottlieb, O., et al. 2019, NatAs, 3, 940

IceCube Collaboration, Aartsen, M. G., Ackermann, M., et al. 2018, Sci, 361 , eaat 1378

Ivezić, Ž, Kahn, S. M., Tyson, J. A., et al. 2019, ApJ, 873, 111

Jin, Z.-P., Covino, S., Liao, N.-H., et al. 2020, NatAs, 4, 77

Jin, Z.-P., Hotokezaka, K., Li, X., et al. 2016, NatCo, 7, 12898

Jin, Z.-P., Li, X., Cano, Z., et al. 2015, ApJL, 811, L22

Jin, Z.-P., Li, X., Wang, H., et al. 2018, ApJ, 857, 128

Kalogera, V., Kim, C., Lorimer, D. R., et al. 2004, ApJL, 614, L137

Kasen, D., Metzger, B., Barnes, J., Quataert, E., \& Ramirez-Ruiz, E. 2017, Natur, 551, 80

Kasliwal, M. M., Anand, S., Ahumada, T., et al. 2020, arXiv:2006.11306

Kasliwal, M. M., Kasen, D., Lau, R. M., et al. 2019, MNRAS, in press

Kasliwal, M. M., Nakar, E., Singer, L. P., et al. 2017, Sci, 358, 1559

Kawaguchi, K., Shibata, M., \& Tanaka, M. 2018, ApJL, 865, L21

Kim, C., Perera, B. B. P., \& McLaughlin, M. A. 2015, MNRAS, 448, 928

Kulkarni, S. R. 2005, arXiv:astro-ph/0510256

Lamb, G. P., Tanvir, N. R., Levan, A. J., et al. 2019, ApJ, 883, 48

Li, L.-X., \& Paczyński, B. 1998, ApJL, 507, L59

Lundquist, M. J., Paterson, K., Fong, W., et al. 2019, ApJL, 881, L26

Luri, X., Brown, A. G. A., Sarro, L. M., et al. 2018, A\&A, 616, A9

Margutti, R., Alexander, K. D., Xie, X., et al. 2018, ApJL, 856, L18

Masci, F. J., Laher, R. R., Rusholme, B., et al. 2019, PASP, 131, 018003

McBrien, O. R., Smartt, S. J., Huber, M. E., et al. 2020, arXiv:2006.10442

Metzger, B. D. 2019, LRR, 23, 1

Metzger, B. D., \& Berger, E. 2012, ApJ, 746, 48

Metzger, B. D., Martínez-Pinedo, G., Darbha, S., et al. 2010, MNRAS, 406, 2650

Miller, A. A., Kulkarni, M. K., Cao, Y., et al. 2017, AJ, 153, 73

Nakar, E. 2019, arXiv:1912.05659

Narayan, R., Paczynski, B., \& Piran, T. 1992, ApJL, 395, L83

Oke, J. B., Cohen, J. G., Carr, M., et al. 1995, PASP, 107, 375

Paczynski, B. 1986, ApJL, 308, L43

pandas development team, T. 2020, pandas-dev/pandas: Pandas, Zenodo, doi:10.5281/zenodo.3509134

Patterson, M. T., Bellm, E. C., Rusholme, B., et al. 2019, PASP, 131, 018001 Perley, D. A. 2019, PASP, 131, 084503

Perley, D. A., Metzger, B. D., Granot, J., et al. 2009, ApJ, 696, 1871

Pian, E., D’Avanzo, P., Benetti, S., et al. 2017, Natur, 551, 67

Planck Collaboration, Abergel, A., Ade, P. A. R., et al. 2014, A\&A, 571, A11 Planck Collaboration, Ade, P. A. R., Aghanim, N., et al. 2016, A\&A, 594, A13

Pol, N., McLaughlin, M., \& Lorimer, D. R. 2019, ApJ, 870, 71

Pol, N., McLaughlin, M., \& Lorimer, D. R. 2020, RNAAS, 4, 22

Ricker, G. R., Winn, J. N., Vanderspek, R., et al. 2015, JATIS, 1, 014003

Rigault, M. 2018, ztfquery, a Python Tool to Access ZTF Data, Zenodo, doi: $10.5281 /$ zenodo. 1345222

Rossi, A., Stratta, G., Maiorano, E., et al. 2020, MNRAS, 493, 3379

Rosswog, S. 2005, ApJ, 634, 1202

Rosswog, S., Sollerman, J., Feindt, U., et al. 2018, A\&A, 615, A132

Sagués Carracedo, A., Bulla, M., Feindt, U., \& Goobar, A. 2020, arXiv:2004. 06137

Salafia, O. S., \& Giacomazzo, B. 2020, arXiv:2006.07376

Schady, P., Xu, D., Heintz, K. E., et al. 2019, GCN, 23632, 1

Schlegel, D. J., Finkbeiner, D. P., \& Davis, M. 1998, ApJ, 500, 525 
Scolnic, D., Kessler, R., Brout, D., et al. 2018, ApJL, 852, L3

Shappee, B. J., Prieto, J. L., Grupe, D., et al. 2014, ApJ, 788, 48

Shappee, B. J., Simon, J. D., Drout, M. R., et al. 2017, Sci, 358, 1574

Siegel, D. M., Barnes, J., \& Metzger, B. D. 2019, Natur, 569, 241

Smartt, S. J., Chambers, K. C., Smith, K. W., et al. 2019, TNSAN, 48, 1

Smartt, S. J., Chen, T. W., Jerkstrand, A., et al. 2017, Natur, 551, 75

Smartt, S. J., Eldridge, J. J., Crockett, R. M., \& Maund, J. R. 2009, MNRAS, 395, 1409

Sonbas, E., Barthelmy, S. D., Beardmore, A. P., et al. 2019, GCN, 23615, 1

Stachie, C., Coughlin, M. W., Christensen, N., \& Muthukrishna, D. 2019, arXiv: 1912.06383

Stein, R., van Velzen, S., Kowalski, M., et al. 2020, arXiv:2005.05340

Tanaka, M., \& Hotokezaka, K. 2013, ApJ, 775, 113

Tanvir, N. R., Levan, A. J., Fruchter, A. S., et al. 2013, Natur, 500, 547

Tanvir, N. R., Levan, A. J., González-Fernández, C., et al. 2017, ApJL, $848, \mathrm{~L} 27$
The Astropy Collaboration 2018, astropy v3.0.5: a Core Python Package for Astronomy, Zenodo, doi:10.5281/zenodo. 2556700

Troja, E., Piro, L., van Eerten, H., et al. 2017, Natur, 551, 71

Troja, E., Ryan, G., Piro, L., et al. 2018, NatCo, 9, 4089

Tutukov, A. V., \& Yungelson, L. R. 1993, MNRAS, 260, 675

Utsumi, Y., Tanaka, M., Tominaga, N., et al. 2017, PASJ, 69, 101

Valenti, S., Sand, D. J., Yang, S., et al. 2017, ApJL, 848, L24

van Eerten, H., Zhang, W., \& MacFadyen, A. 2010, ApJ, 722, 235

van Roestel, J., Groot, P. J., Kupfer, T., et al. 2019, MNRAS, 484, 4507

Vieira, N., Ruan, J. J., Haggard, D., et al. 2020, ApJ, 895, 96

Watson, A. M., Butler, N. R., Lee, W. H., et al. 2020, MNRAS, 492, 5916

Yang, B., Jin, Z.-P., Li, X., et al. 2015, NatCo, 6, 7323

Yang, S., Valenti, S., Cappellaro, E., et al. 2017, ApJL, 851, L48

Yao, Y., Miller, A. A., Kulkarni, S. R., et al. 2019, ApJ, 886, 152

Yurkov, V., Gabovich, A., Sergienko, Y., et al. 2019, GCN, 23614, 1

Zackay, B., Ofek, E. O., \& Gal-Yam, A. 2016, ApJ, 830, 27 